\title{
Synthesis, Reactions, Spectral Behavior and Biological Evaluation of Some New D-Glucopyranoside Derivatives as Potential Antimicrobial Agents
}

\author{
Potansiyel Antimikrobiyal Ajanlar Olarak Bazı Yeni \\ D-Glukopiranozit Türevlerinin Sentezi, Tepkimeleri, Spektral \\ Davranışları ve Biyolojik Değerlendirmesi
}

Research Article

Sarkar Mohammad Abe Kawsar ${ }^{1,3 *}$, Refat Asma1, Khaleda Mymona', Mohammad Abul Manchur², Imtiaj Hasan $^{3}$, Yasuhiro Ozeki ${ }^{4}$

'Laboratory of Carbohydrate and Protein Chemistry, Department of Chemistry, Faculty of Science, University of Chittagong, Chittagong, Bangladesh. 2Department of Microbiology, Faculty of Biological Science, University of Chittagong, Chittagong, Bangladesh.

${ }^{3}$ Department of Biochemistry and Molecular Biology, University of Rajshahi, Rajshahi, Bangladesh.

${ }^{4}$ Laboratory of Glycobiology and Marine Biochemistry, Department of Life and Environmental System Science, Graduate School of NanoBio Sciences, Yokohama City University, 22-2 Seto, Kanazawaku, Yokohama, Japan.

\section{A B S TR ACT}

\begin{abstract}
In the present investigation, a new series of methyl 6-0-cinnamoyl- $\alpha$-D-glucopyranoside derivatives were synthesized by the reaction of methyl $\alpha$-D-glucopyranoside (1) with various non-traditional acylating agents in pyridine at $-5^{\circ} \mathrm{C}$. For this purpose, firstly, selective cinnamoylation of methyl $\alpha$-D-glucopyranoside (1) has been carried out by the direct acylation method and afforded the methyl 6-0-cinnamoyl- $\alpha$-D-glucopyranoside (2) in good yield. Secondly, in order to obtain newer products for antimicrobial screening studies, the 6-0-cinnamoyl derivative was further transformed to a series of 2,3,4-tri-0-acyl derivatives (4-14) containing a wide variety of functionalities in a single molecular framework. The structures of the newly synthesized compounds were characterized by IR, NMR and physicochemical properties analyses. All the compounds were evaluated for their in vitro antibacterial and antifungal activities using the disc diffusion and food poisoning methods. The study revealed that the compound methyl 6-0-cinnamoyl-2,3,4-tri-0-decanoyl- $\alpha$-D-glucopyranoside (7) showed the highest inhibition activity against both Gram (+) B. subtilis and Gram (-) P. aeruginosa microorganisms. The tested compound methyl 6-0-cinnamoyl-2,3,4-tri-0-lauroyl- $\alpha$-D-glucopyranoside (8) exhibited maximum mycelial growth inhibition against A. niger fungi. The acylated derivatives were found to be more effective against the fungal strains than those of the bacterial pathogens. This is the first report of the antimicrobial activity of the selected chemicals against the selected pathogens.
\end{abstract}

Key Words

D-Glucopyranoside, acylation, structure, spectroscopy, antibacterial, antifungal.

\section{ÖZET}

B u çalışmada, metil 6-0-sinamoil- $\alpha$-D-glukopiranozid türevlerinin yeni bir serisi, piridinde çeşitli açilleyici ajanlarla, metil $\alpha$-D-glukopiranozid (1) tepkimesi ile $-5^{\circ} \mathrm{C}$ 'da sentezlendi. Bu amaçla ilk olarak metil $\alpha$-D-glukopiranosid in seçici sinamalasyonu, doğrudan asilasyon yöntemi ile gerçekleştirildi ve iyi bir verimle metil 6-0-sinamoil- $\alpha$-D-glukopiranozid elde edildi. İkinci olarak, antimikrobiyal tarama çalışmaları için yeni ürünler elde etmek amacıyla, 6 sinamoil türevleri tek bir molekül çerçevesinde 2,3,4-tri-0-asil türevlerini içeren geniş fonkisyonel gruplara dönüştürüldü. Yeni sentezlenen bileşiklerin yapıları IR ve NMR ile karakterize edildi. Tüm bileşikler, gıda zehirlenmesi ve disk difüzyon yöntemleri kullanılarak in vitro ortamda antibakteriyel ve antifungal aktiviteleri açısından değerlendirildi. Çalışmada metil 6-0-sinamoil-2,3,4-tri-0-dekanoil$\alpha$-D-glukopiranosid bileşiği hem Gram (+) B. subtilise hem de Gram (-) P. aeruginosa mikroorganizmasına karşı yüksek inhibisyon etkisi gösterdi. Test bileşiği, metil 6-0-sinamoil-2,3,4-tri-0-lauroil- $\alpha$-D-glukopiranosid A ya karşı maksimum misel büyüme inhibisyonu sergiledi. Açillenmiş türevlerin fungal hattına karşı olan etkisinin bakteriyel patojenlere göre daha yüksek olduğu bulundu. Bu, seçilen patojenlere karşı seçilmiş kimyasalların antimikrobiyal etkisini gösteren ilk raporudur.

\section{Anahtar Kelimeler}

D-glukopiranozid, açilasyon, yapı, spektroskopi, antibakteriyel, antifungal.

Article History: Received: Oct 15, 2015; Revised: Nov 19, 2015; Accepted: Nov 20, 2015; Available Online: Dec $30,2015$. DOI: 10.15671/HJBC.20154315990

Correspondence to: S.M.A. Kawsar, Department of Chemistry, Faculty of Science, University of Chittagong, Bangladesh. Tel: +88017 62717081 


\section{INTRODUCTION}

Carbohydrates are an important class of macromolecules that serve various roles in biological systems [1,2]. Besides the commonlyknownfunctionsformetabolicenergy (saccharides, such as starch and glycogen, provide fuel for biological systems) and for mechanical support (saccharides, such as cellulose, are the essential building block of cell walls), carbohydrates can play role in signal transduction, immune response, cell trafficking, cell adhesion and cell-cell interaction. So, the study of carbohydrates is one of the most exciting fields of organic chemistry for chemists. An important part of a reaction sequence in carbohydrate chemistry is to protect the different hydroxyl groups that are not taking part in a desired reaction. For protection of hydroxyl groups, a wide variety of methods have already been developed [3-7]. Of these, acylation by the direct acylation method was found to be one of the most encouraging methods [8].

Antimicrobial agents inhibit or kill the growth of microorganisms such as bacteria or fungus. By means of antimicrobial drug discovery, it is believed that microbial infections will end up. However, rapid increases of microorganism originated diseases make it difficult to happen. Furthermore, senseless usage of antimicrobials exposed another big problem, drug resistance [9, 10]. As a result of this, the need for the synthesis and development of new antimicrobial agents has emerged [11-13].

During the last few decades, considerable works have been done in the field of biological evaluation of various chemical compounds [14]. Carbohydrates, especially- acylated glycoses and glycosides, are very important due to their effective biological activity. It is known that if an active nucleus is linked to another nucleus, the resultant molecule may possess greater potential for biological activity [15]. From literature survey, it was revealed [16] that a large number of biological compounds possess aromatic, heteroaromatic and acyl substituents. Nitrogen $(\mathrm{N})$, sulphur (S) and halogen (X) containing substituents are also known to enhance the biological activity of the parent compound [16]. A wide variety of heterocyclic compounds bearing this moiety has been reported as significant olecules with broad spectrum of biological activities such as antimicrobial $[17,18]$, anticancer $[19,20]$, antitubercular $[21,22]$, anti-inflammatory [23], analgesic [24], and antiviral activities [25].

Over the last few years, researchers in our carbohydrate \& protein chemistry laboratory carried out selective acylation of monosaccharide derivatives [26-30] and also biological evaluation of the synthesized compounds [31-34]. It was observed that the combination of two or more acyl substituents in a single molecular framework enhances the biological activity many fold than their parent nuclei. For example, some acylated derivatives of $D$-glucopyranose were found more active than those of the standard antibiotics $[35,36]$.

Encouraged by our own findings and also literature reports, we synthesized a series of D-glucopyranoside derivatives (Scheme 1 and Table 1) incorporating a cinnamoyl group as well as a wide variety of other probable biologically active components to the D-glucose moiety. Antibacterial and antifungal activities of these newly synthesized compounds were evaluated using a variety of human pathogenic bacterial and phyto-pathogenic fungal strains and the experiments are reported here.

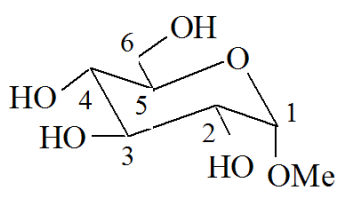

\section{EXPERIMENTAL}

\section{Materials and chemicals}

Infrared (IR) spectra were recorded on a FourierTransform Infrared (FTIR) spectrophotometer (SHIMADZU, IR Prestige-21) using the $\mathrm{KBr} / \mathrm{CHCl}_{3}$ technique at the Department of Chemistry, University of Chittagong, Bangladesh. ${ }^{1} \mathrm{H}-\mathrm{NMR}$ $\left(400 \mathrm{MHz}\right.$ ) spectra were recorded using $\mathrm{CDCl}_{3}$ as a solvent. Chemical shifts were reported in $\alpha$ unit (ppm) using tetramethyl silane (TMS) as the internal standard and $J$ values are given in $\mathrm{Hz}$ with a Bruker DPX-400 spectrometer 


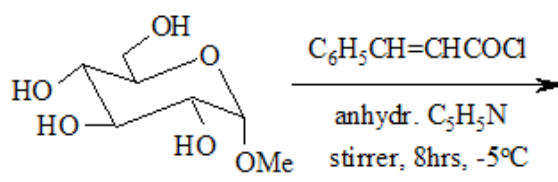

1

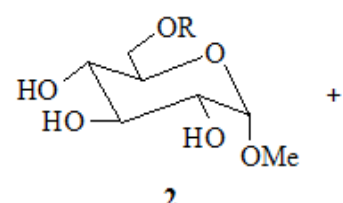

2

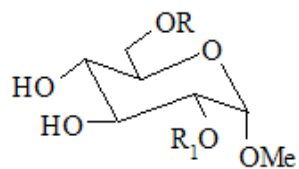

3

$$
\left(\mathrm{R}=\mathrm{C}_{6} \mathrm{H}_{5} \mathrm{CH}=\mathrm{CHCO}-\right) \quad\left(\mathrm{R}=\mathrm{R}_{1}=\mathrm{C}_{6} \mathrm{H}_{5} \mathrm{CH}=\mathrm{CHCO}-\right)
$$

Separated by

Chromatographic techniques

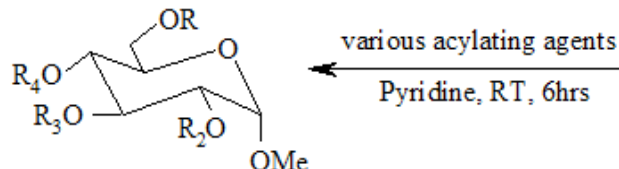

4-14

(2,3,4-Tri- $O$-acylated products)

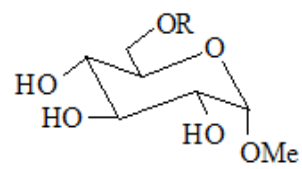

2

(6-O-cinnam oyl derivative)

Schme 1. Schematic reaction path of D-glucopyranoside derivatives [2-14].

Table 1. Substituted acyl residues for the synthesis of methyl $\alpha$-D-glucopyranoside derivatives.

\begin{tabular}{cccc}
\hline Compound no & \multicolumn{1}{c}{$\mathbf{R}$} & $\mathbf{R}_{2}=\mathbf{R}_{3}=\mathbf{R}_{4}$ & \multicolumn{1}{c}{ Mol. Formula } \\
\hline 2 & $\mathrm{C}_{6} \mathrm{H}_{5} \mathrm{CH}=\mathrm{CHCO}-$ & $\mathrm{H}$ & $\mathrm{C}_{16} \mathrm{H}_{20} \mathrm{O}_{7}$ \\
\hline 3 & $\mathrm{C}_{6} \mathrm{H}_{5} \mathrm{CH}=\mathrm{CHCO}-$ & $\mathrm{R}_{2}=\mathrm{C}_{6} \mathrm{H}_{5} \mathrm{CH}=\mathrm{CHCO}-\mathrm{R}_{3}=\mathrm{R}_{4}$ & $\mathrm{C}_{25} \mathrm{H}_{26} \mathrm{O}_{8}$ \\
\hline 4 & $\mathrm{C}_{6} \mathrm{H}_{5} \mathrm{CH}=\mathrm{CHCO}-$ & $\mathrm{CH}_{3} \mathrm{CO}-$ & $\mathrm{C}_{22} \mathrm{H}_{26} \mathrm{O}_{10}$ \\
\hline 5 & $\mathrm{C}_{6} \mathrm{H}_{5} \mathrm{CH}=\mathrm{CHCO}-$ & $\mathrm{CH}_{3}\left(\mathrm{CH}_{2}\right)_{3} \mathrm{CO}-$ & $\mathrm{C}_{31} \mathrm{H}_{44} \mathrm{O}_{10}$ \\
\hline 6 & $\mathrm{C}_{6} \mathrm{H}_{5} \mathrm{CH}=\mathrm{CHCO}-$ & $\mathrm{CH}_{3}\left(\mathrm{CH}_{2}\right)_{4} \mathrm{CO}-$ & $\mathrm{C}_{34} \mathrm{H}_{50} \mathrm{O}_{10}$ \\
\hline 7 & $\mathrm{C}_{6} \mathrm{H}_{5} \mathrm{CH}=\mathrm{CHCO}-$ & $\mathrm{CH}_{3}\left(\mathrm{CH}_{2}\right)_{8} \mathrm{CO}-$ & $\mathrm{C}_{46} \mathrm{H}_{74} \mathrm{O}_{10}$ \\
\hline 8 & $\mathrm{C}_{6} \mathrm{H}_{5} \mathrm{CH}=\mathrm{CHCO}-$ & $\mathrm{CH}_{3}\left(\mathrm{CH}_{2}\right)_{10} \mathrm{CO}-$ & $\mathrm{C}_{52} \mathrm{H}_{86} \mathrm{O}_{10}$ \\
\hline 9 & $\mathrm{C}_{6} \mathrm{H}_{5} \mathrm{CH}=\mathrm{CHCO}-$ & $\mathrm{CH}_{3}\left(\mathrm{CH}_{2}\right)_{12} \mathrm{CO}-$ & $\mathrm{C}_{58} \mathrm{H}_{98} \mathrm{O}_{10}$ \\
\hline 10 & $\mathrm{C}_{6} \mathrm{H}_{5} \mathrm{CH}=\mathrm{CHCO}-$ & $\mathrm{CH}_{3}\left(\mathrm{CH}_{2}\right)_{14} \mathrm{CO}-$ & $\mathrm{C}_{64} \mathrm{H}_{110} \mathrm{O}_{10}$ \\
\hline 11 & $\mathrm{C}_{6} \mathrm{H}_{5} \mathrm{CH}=\mathrm{CHCO}-$ & $\left(\mathrm{CH}_{3}\right)_{3} \mathrm{CCO}^{-}$ & $\mathrm{C}_{31} \mathrm{H}_{44} \mathrm{O}_{10}$ \\
\hline 12 & $\mathrm{C}_{6} \mathrm{H}_{5} \mathrm{CH}=\mathrm{CHCO}-$ & $\mathrm{CH}_{3} \mathrm{SO}_{2}^{-}$ & $\mathrm{C}_{19} \mathrm{H}_{26} \mathrm{~S}_{3} \mathrm{O}_{13}$ \\
\hline 13 & $\mathrm{C}_{6} \mathrm{H}_{5} \mathrm{CH}=\mathrm{CHCO}-$ & $\mathrm{C}_{6} \mathrm{H}_{5} \mathrm{SO}_{2}^{-}$ & $\mathrm{C}_{35} \mathrm{H}_{32} \mathrm{~S}_{3} \mathrm{O}_{13}$ \\
\hline 14 & $\mathrm{C}_{6} \mathrm{H}_{5} \mathrm{CH}=\mathrm{CHCO}-$ & $4-\mathrm{Cl}_{6} \mathrm{C}_{6} \mathrm{H}_{4} \mathrm{CO}-$ & $\mathrm{C}_{37} \mathrm{H}_{20} \mathrm{C}_{13} \mathrm{O}_{10}$ \\
\hline
\end{tabular}

at the Bangladesh Council of Scientific and Industrial Research (BCSIR) Laboratories, Dhaka, Bangladesh. Evaporations were carried out under reduced pressure using VV-1 type vacuum rotary evaporator (Germany) with a bath temperature below $40^{\circ} \mathrm{C}$. Melting points $(\mathrm{mp})$ were determined using an electro-thermal melting point apparatus (England) and are uncorrected. Thin layer chromatography (TLC) was performed on
Kieselgel $\mathrm{GF}_{254}$ and spots were detected by spraying the plates with $1 \% \mathrm{H}_{2} \mathrm{SO}_{4}$ followed by heating the plates at $150^{\circ} \mathrm{C}-200^{\circ} \mathrm{C}$ until color appeared. Column chromatography was carried out with silica gel $G_{60}(100-200$ mesh). Solvent system employed for TLC analyses was methanol, ethyl acetate, chloroform and $n$-hexane. All solvents and reagents were purchased from Sigma-Aldrich and Merck unless reported 
otherwise and used without further purifications. The reaction pathways have been summarized in Scheme 1.

\section{Reactions and synthesis \\ Reaction of methyl- $\alpha$-D-glucopyranoside (1) with cinnamoyl chloride}

A suspension of methyl- $\alpha$-D-glucopyranoside (1) $(102 \mathrm{mg}, 0.52 \mathrm{mmol})$ in dry pyridine $(3 \mathrm{ml})$ was cooled to $-5^{\circ} \mathrm{C}$ in an ice bath whereupon cinnamoyl chloride (90 mg, 1.1 molar eq.) was added to it. The reaction mixture was continuously stirred for 8 hours at $0^{\circ} \mathrm{C}$ temperature and then the reaction mixture was stand for overnight at room temperature with continuous stirring. The progress of the reaction was monitored by t.l.c which indicated the formation of two products, the slower moving component being the major one. A few pieces of ice was added to the flask and then extracted the product mixture with chloroform $(30 \times 10 \mathrm{ml})$. The combined chloroform layer was washed successively with dilute hydrochloric acid $(10 \%)$, saturated aqueous $\mathrm{NaHCO}_{3}$ solution and distilled water. The chloroform layer was dried with anhydrous $\mathrm{MgSO}_{4}$, filtered and the filtrate was concentrated under reduced pressure to leave a syrup. The syrup was passed through a silica gel column chromatography and eluted with methanol-chloroform. Initial elution provided the faster moving component (3). Further elution with $\mathrm{CH}_{3} \mathrm{OH}-\mathrm{CHCl}_{3}$ (1:16) furnished the slower moving component, 6-O-cinnamoyl derivative (2). This compound was sufficiently pure for its use as the starting material for the acylation reactions as reported in this manuscript.

\section{Methyl 6-0-cinnamoyl- $\alpha$-D-glucopyranoside 2}

Yield (55 mg, 53\%) as colourless needles, m.p. $58-60^{\circ} \mathrm{C}, \mathrm{R}_{\mathrm{f}} \mathrm{O} .51\left(\mathrm{CH}_{3} \mathrm{OH}: \mathrm{CHCl} 3,1: 16\right)$. Anal. calcd. for $\mathrm{C}_{16} \mathrm{H}_{20} \mathrm{O}_{7}$ : C, 59.26; $\mathrm{H}, 6.17 \%$. Found: $\mathrm{C}, 59.31 ; \mathrm{H}$, 6.22\%. FTIR 3385-3525 (br -OH), $1728(-\mathrm{C}=0)$, $1638\left(-\mathrm{CH}=\mathrm{CH}^{-}\right) \mathrm{cm}^{-1} .{ }^{1} \mathrm{H}$ NMR $\left(\mathrm{CDCl}_{3}, \mathrm{ppm}\right): \delta_{\mathrm{H}}=$ $7.69(1 \mathrm{H}, \mathrm{d}, J=16.0 \mathrm{~Hz}, \mathrm{PhCH}=\mathrm{CHCO}-), 7.47(2 \mathrm{H}, \mathrm{m}$, Ar-H), $7.33(3 \mathrm{H}, \mathrm{m}, \mathrm{Ar}-\mathrm{H}), 6.46(1 \mathrm{H}, \mathrm{d}, J=16.0 \mathrm{~Hz}$, $\mathrm{PhCH}=\mathrm{CHCO}-), 4.75(1 \mathrm{H}, \mathrm{d}, J=3.5 \mathrm{~Hz}, \mathrm{H}-1), 4.56$ $(1 \mathrm{H}, \mathrm{dd}, J=5.1$ and $12.2 \mathrm{~Hz}, \mathrm{H}-6 \mathrm{a}), 4.18(1 \mathrm{H}, \mathrm{dd}, J=$ 2.1 and $12.1 \mathrm{~Hz}, \mathrm{H}-6 \mathrm{~b}), 3.79(1 \mathrm{H}, \mathrm{t}, J=9.2 \mathrm{~Hz}, \mathrm{H}-3)$, $3.56(1 \mathrm{H}, \mathrm{t}, J=9.6 \mathrm{~Hz}, \mathrm{H}-4), 3.43(1 \mathrm{H}, \mathrm{dd}, J=3.5$ and $10.0 \mathrm{~Hz}, \mathrm{H}-2), 3.38(1 \mathrm{H}, \mathrm{ddd}, J=2.8,9.8$ and $12.7 \mathrm{~Hz}, \mathrm{H}-5), 3.25\left(3 \mathrm{H}, \mathrm{s}, 1-\mathrm{OCH}_{3}\right)$.

\section{Methyl2,6-di-o-cinnamoyl- $\alpha$-D- glucopyranoside 3}

Yield ( $27 \mathrm{mg}, 28 \%$ ) as colourless pasty mass, $R_{f}$ $0.51\left(\mathrm{CH}_{3} \mathrm{OH}: \mathrm{CHCl}_{3}, 1: 15\right)$. Anal. calcd. for $\mathrm{C}_{25} \mathrm{H}_{26} \mathrm{O}_{8}$ : C, 66.08; $H, 5.73 \%$. Found: $C, 66.11 ; H, 5.77 \%$. FTIR 3360-3510 (br -OH), $1735(-\mathrm{C}=0), 1631(-\mathrm{CH}=\mathrm{CH}-)$ $\mathrm{cm}-1 .{ }^{1} \mathrm{H}$ NMR $\left(\mathrm{CDCl}_{3}, \mathrm{ppm}\right): \delta \mathrm{H}=7.72,7.68(2 \times 1 \mathrm{H}$, $2 \times \mathrm{d}, J=16.0 \mathrm{~Hz}, 2 \times \mathrm{PhCH}=\mathrm{CHCO}-), 7.45(4 \mathrm{H}, \mathrm{m}, \mathrm{Ar}-$ $\mathrm{H}), 7.34(6 \mathrm{H}, \mathrm{m}, \mathrm{Ar}-\mathrm{H}), 6.48,6.44(2 \times 1 \mathrm{H}, 2 \times \mathrm{d}, \mathrm{J}=$ $16.1 \mathrm{~Hz}, 2 \times \mathrm{PhCH}=\mathrm{CHCO}-), 4.98(1 \mathrm{H}, \mathrm{d}, J=3.6 \mathrm{~Hz}$, $\mathrm{H}-1), 4.88(1 \mathrm{H}, \mathrm{dd}, J=3.6$ and $10.2 \mathrm{~Hz}, \mathrm{H}-2), 4.57$ $(1 \mathrm{H}, \mathrm{dd}, J=5.2$ and $12.1 \mathrm{~Hz}, \mathrm{H}-6 \mathrm{a}), 4.44(1 \mathrm{H}, \mathrm{dd}, J=$ 2.2 and $12.1 \mathrm{~Hz}, \mathrm{H}-6 \mathrm{~b}), 4.11(1 \mathrm{H}, \mathrm{t}, J=9.4 \mathrm{~Hz}, \mathrm{H}-3)$, $3.86(1 \mathrm{H}, \mathrm{t}, J=9.7 \mathrm{~Hz}, \mathrm{H}-4), 3.59(1 \mathrm{H}, \mathrm{ddd}, J=3.0$, 9.9 and $12.8 \mathrm{~Hz}, \mathrm{H}-5), 3.36\left(3 \mathrm{H}, \mathrm{s}, 1-\mathrm{OCH}_{3}\right)$.

\section{General procedure for the synthesis of 6-0-cinnamoyl- $\alpha$-D-glucopyranoside derivatives 4-14}

A stirred cooled $\left(-5^{\circ} \mathrm{C}\right)$ solution of methyl 6-O-cinnamoyl- $\alpha$-D-glucopyranoside (2) $\quad 69$ $\mathrm{mg}, 0.22 \mathrm{mmol})$, in dry pyridine $(3 \mathrm{ml})$, acetic anhydride $(0.10 \mathrm{ml}, 5$ molar eq.) was added. The reaction mixture was continuously stirred for $8 \mathrm{hrs}$ at same temperature for overnight. The progress of the reaction was monitored by t.l.c (ethyl acetate-n-hexane, 1:4) which indicated the complete conversion of the starting material into faster moving product. Work-up as usual and purification by passage through a silica gel column chromatography with ethyl acetate-nhexane, yielded the acetyl derivative (4) as a semi solid mass, which resisted crystallization. Similar reaction and purification procedure was applied to prepare compound 5-14.

\section{Methyl 2,3,4-tri-0-acetyl-6-0-cinnamoyl- $\alpha-$ D-glucopyranoside 4}

Yield (50 mg, 72\%) as white needles, m.p. 65$67^{\circ} \mathrm{C}, \mathrm{R}_{\mathrm{f}} \mathrm{O} .50$ (EtOAc: $\mathrm{nC}_{6} \mathrm{H}_{14}, 1: 4$ ). Anal. calcd. for $\mathrm{C}_{22} \mathrm{H}_{26} \mathrm{O}_{10}: \mathrm{C}, 58.67 ; \mathrm{H}, 5.78 \%$. Found: $\mathrm{C}, 58.71 ; \mathrm{H}$, $5.83 \%$. FTIR $1712,1778(-\mathrm{C}=0), 1628(-\mathrm{CH}=\mathrm{CH}-)$ $\mathrm{cm}^{-1} .{ }^{1} \mathrm{H}$ NMR $\left(\mathrm{CDCl}_{3}, \mathrm{ppm}\right): \delta_{\mathrm{H}}=7.72(1 \mathrm{H}, \mathrm{d}, J=16.1$ $\mathrm{Hz}, \mathrm{PhCH}=\mathrm{CHCO}-), 7.52(2 \mathrm{H}, \mathrm{m}, \mathrm{Ar}-\mathrm{H}), 7.38(3 \mathrm{H}, \mathrm{m}$, Ar-H), $6.42(1 \mathrm{H}, \mathrm{d}, J=16.0 \mathrm{~Hz}, \mathrm{PhCH}=\mathrm{CHCO}-), 5.57$ $(1 \mathrm{H}, \mathrm{d}, J=3.6 \mathrm{~Hz}, \mathrm{H}-1), 5.46(1 \mathrm{H}, \mathrm{dd}, J=3.6$ and 10.1 $\mathrm{Hz}, \mathrm{H}-2), 5.02(1 \mathrm{H}, \mathrm{t}, J=9.7 \mathrm{~Hz}, \mathrm{H}-3), 4.99(1 \mathrm{H}, \mathrm{t}, J=$ $9.7 \mathrm{~Hz}, \mathrm{H}-4), 4.89(1 \mathrm{H}, \mathrm{m}, \mathrm{H}-6 \mathrm{a}), 4.32(1 \mathrm{H}, \mathrm{t}, J=10.2$ $\mathrm{Hz}, \mathrm{H}-6 \mathrm{~b}), 4.08(1 \mathrm{H}, \mathrm{m}, \mathrm{H}-5), 3.43(3 \mathrm{H}, \mathrm{s}, 1-\mathrm{OCH} 3)$, $2.05,2.01,1.98\left(3 \times 3 \mathrm{H}, 3 \times \mathrm{S}, 3 \times \mathrm{CH}_{3} \mathrm{CO}-\right)$. 


\section{Methyl 6-0-cinnamoyl-2,3,4-tri-0-pentanoyl- $\alpha$-D-glucopyranoside 5}

Yield (48 mg, 88\%) as colourless pasty mass, $R_{f}$ 0.52 (EtOAc: $\mathrm{nC}_{6} \mathrm{H}_{14}, 1: 5$ ). Anal. calcd. for $\mathrm{C}_{31} \mathrm{H}_{44} \mathrm{O}_{10}$ : C, 64.58; $\mathrm{H}, 7.64 \%$. Found: $\mathrm{C}, 64.66 ; \mathrm{H}, 7.71 \%$. FTIR $1758 \quad(-\mathrm{C}=0), 1630 \quad(-\mathrm{CH}=\mathrm{CH}-) \quad \mathrm{cm}^{-1} \cdot{ }^{1} \mathrm{H}$ $\operatorname{NMR}\left(\mathrm{CDCl}_{3}, \mathrm{ppm}\right): \delta_{\mathrm{H}}=7.72(1 \mathrm{H}, \mathrm{d}, J=16.2 \mathrm{~Hz}$, $\mathrm{PhCH}=\mathrm{CHCO}-), 7.52(2 \mathrm{H}, \mathrm{m}, \mathrm{Ar}-\mathrm{H}), 7.38(3 \mathrm{H}, \mathrm{m}$ $\mathrm{Ar}-\mathrm{H}), 6.44(1 \mathrm{H}, \mathrm{d}, \mathrm{J}=16.2 \mathrm{~Hz}, \mathrm{PhCH}=\mathrm{CHCO}-), 5.33$ $(1 \mathrm{H}, \mathrm{d}, J=3.6 \mathrm{~Hz}, \mathrm{H}-1), 4.95(1 \mathrm{H}, \mathrm{dd}, J=3.6$ and $10.0 \mathrm{~Hz}, \mathrm{H}-2), 4.85(1 \mathrm{H}, \mathrm{t}, J=9.6 \mathrm{~Hz}, \mathrm{H}-3), 4.62(1 \mathrm{H}$ $\mathrm{t}, J=9.5 \mathrm{~Hz}, \mathrm{H}-4), 4.35(1 \mathrm{H}, \mathrm{dd}, J=5.0$ and 12.0 $\mathrm{Hz}, \mathrm{H}-6 \mathrm{a}), 4.12(1 \mathrm{H}, \mathrm{dd}, J=2.1$ and $12.2 \mathrm{~Hz}, \mathrm{H}-6 \mathrm{~b})$, $3.57(1 \mathrm{H}, \mathrm{ddd}, J=2.9,9.9$ and $12.8 \mathrm{~Hz}, \mathrm{H}-5), 3.39$ $\left(3 \mathrm{H}, \mathrm{s}, 1-\mathrm{OCH}_{3}\right), 2.31\left\{6 \mathrm{H}, \mathrm{m}, 3 \times \mathrm{CH}_{3}\left(\mathrm{CH}_{2}\right)_{2} \mathrm{CH}_{2} \mathrm{CO}-\right\}$, $1.61\left\{6 \mathrm{H}, \quad \mathrm{m}, \quad 3 \times \mathrm{CH}_{3} \mathrm{CH}_{2} \mathrm{CH}_{2} \mathrm{CH}_{2} \mathrm{CO}-\right\}, \quad 1.33$ $\left\{6 \mathrm{H}, \quad \mathrm{m}, 3 \times \mathrm{CH}_{3} \mathrm{CH}_{2}\left(\mathrm{CH}_{2}\right)_{2} \mathrm{CO}-\right\}, 0.88\{9 \mathrm{H}, \mathrm{m}$, $\left.3 \times \mathrm{CH}_{3}\left(\mathrm{CH}_{2}\right)_{3} \mathrm{CO}-\right\}$.

\section{Methyl 6-0-cinnamoyl-2,3,4-tri-0-hexanoyl- $\alpha$-D-glucopyranoside 6}

Yield (63 mg, 90\%) as colourless needles, m.p. $46-48^{\circ} \mathrm{C}, \mathrm{R}_{\mathrm{f}} \mathrm{O} .50$ (EtOAc: $\mathrm{nC}_{6} \mathrm{H}_{14}, 1: 7$ ). Anal. calcd. for $\mathrm{C}_{34} \mathrm{H}_{50} \mathrm{O}_{10}: \mathrm{C}, 66.01 ; \mathrm{H}, 8.09 \%$. Found: $\mathrm{C}_{\text {, }}$ 66.06; H, 8.14\%. FTIR 1775 (-CO), $1622(-\mathrm{CH}=\mathrm{CH}-)$ $\mathrm{cm}-1 .{ }^{1} \mathrm{H}$ NMR $\left(\mathrm{CDCl}_{3}, \mathrm{ppm}\right): \delta_{\mathrm{H}}=7.70(1 \mathrm{H}, \mathrm{d}, J=16.0$ $\mathrm{Hz}, \mathrm{PhCH}=\mathrm{CHCO}-), 7.51(2 \mathrm{H}, \mathrm{m}, \mathrm{Ar}-\mathrm{H}), 7.37(3 \mathrm{H}$ $\mathrm{m}, \mathrm{Ar}-\mathrm{H}), 6.44(1 \mathrm{H}, \mathrm{d}, \mathrm{J}=16.0 \mathrm{~Hz}, \mathrm{PhCH}=\mathrm{CHCO}-)$, $5.51(1 \mathrm{H}, \mathrm{d}, J=3.7 \mathrm{~Hz}, \mathrm{H}-1), 5.12(1 \mathrm{H}, \mathrm{dd}, J=3.6$ and $10.1 \mathrm{~Hz}, \mathrm{H}-2), 4.95(1 \mathrm{H}, \mathrm{t}, J=9.6 \mathrm{~Hz}, \mathrm{H}-3)$, $4.89(1 \mathrm{H}, \mathrm{t}, J=9.7 \mathrm{~Hz}, \mathrm{H}-4), 4.32(1 \mathrm{H}, \mathrm{m}, \mathrm{H}-6 \mathrm{a})$, $4.28(1 \mathrm{H}, \mathrm{m}, \mathrm{H}-6 \mathrm{~b}), 4.05(1 \mathrm{H}, \mathrm{m}, \mathrm{H}-5), 3.43(3 \mathrm{H}$ $\left.\mathrm{s}, 1-\mathrm{OCH}_{3}\right), 2.26\left\{6 \mathrm{H}, \mathrm{m}, 3 \times \mathrm{CH}_{3}\left(\mathrm{CH}_{2}\right) 3 \mathrm{CH}_{2} \mathrm{CO}-\right\}_{1}$ $1.60\left\{6 \mathrm{H}, \quad \mathrm{m}, \quad 3 \times \mathrm{CH}_{3}\left(\mathrm{CH}_{2}\right) 2 \mathrm{CH}_{2} \mathrm{CH}_{2} \mathrm{CO}\right\}, \quad 1.24$ $\left\{12 \mathrm{H}, \mathrm{m}, 3 \times \mathrm{CH}_{3}\left(\mathrm{CH}_{2}\right) 2 \mathrm{CH}_{2} \mathrm{CH}_{2} \mathrm{CO}-\right\}, 0.87\{9 \mathrm{H}, \mathrm{m}$, $\left.3 \times \mathrm{CH}_{3}\left(\mathrm{CH}_{2}\right)_{4} \mathrm{CO}-\right\}$.

\section{Methyl 6-0-cinnamoyl-2,3,4-tri-0-decanoyl- $\alpha$-D-glucopyranoside 7}

Yield (108 mg, 86\%) as white semi-solid, $R_{f} 0.53$ (EtOAc: $\mathrm{nC}_{6} \mathrm{H}_{14}, 1: 5$ ). Anal. calcd. for $\mathrm{C}_{46} \mathrm{H}_{74} \mathrm{O}_{10}$ : C 70.23; $\mathrm{H}, 9.41 \%$. Found: $\mathrm{C}, 70.29 ; \mathrm{H}, 9.47 \%$. FTIR $1688(-\mathrm{C}=0), 1642(-\mathrm{CH}=\mathrm{CH}-) \mathrm{cm}^{-1} .{ }^{1} \mathrm{H}$ NMR $\left(\mathrm{CDCl}_{3^{\prime}}\right.$ ppm): $\delta \mathrm{H}=7.68(1 \mathrm{H}, \mathrm{d}, J=15.9 \mathrm{~Hz}, \mathrm{PhCH}=\mathrm{CHCO}-)$ $7.52(2 \mathrm{H}, \mathrm{m}, \mathrm{Ar}-\mathrm{H}), 7.38(3 \mathrm{H}, \mathrm{m}, \mathrm{Ar}-\mathrm{H}), 6.45(1 \mathrm{H}, \mathrm{d}$, $J=16.0 \mathrm{~Hz}, \mathrm{PhCH}=\mathrm{CHCO}-), 5.22(1 \mathrm{H}, \mathrm{d}, J=3.5 \mathrm{~Hz}$, $\mathrm{H}-1), 5.07(1 \mathrm{H}, \mathrm{dd}, J=3.7$ and $10.1 \mathrm{~Hz}, \mathrm{H}-2), 4.85$ $(1 \mathrm{H}, \mathrm{t}, J=9.6 \mathrm{~Hz}, \mathrm{H}-3), 4.77(1 \mathrm{H}, \mathrm{t}, J=9.6 \mathrm{~Hz}, \mathrm{H}-4)$ $4.13(1 \mathrm{H}, \mathrm{dd}, J=4.8$ and $10.0 \mathrm{~Hz}, \mathrm{H}-6 \mathrm{a}), 3.98(1 \mathrm{H}$ $\mathrm{t}, J=10.0 \mathrm{~Hz}, \mathrm{H}-6 \mathrm{~b}), 3.87(1 \mathrm{H}, \mathrm{m}, \mathrm{H}-5), 3.35(3 \mathrm{H}$, $\left.\mathrm{s}, 1-\mathrm{OCH}_{3}\right), 2.34\left\{6 \mathrm{H}, \mathrm{m}, 3 \times \mathrm{CH}_{3}\left(\mathrm{CH}_{2}\right) 7 \mathrm{CH}_{2} \mathrm{CO}-\right\}$, $1.62\left\{6 \mathrm{H}, \quad \mathrm{m}, \quad 3 \times \mathrm{CH}_{3}\left(\mathrm{CH}_{2}\right) 6 \mathrm{CH}_{2} \mathrm{CH}_{2} \mathrm{CO}-\right\}, 1.23$ $\left\{36 \mathrm{H}, \mathrm{m}, 3 \times \mathrm{CH}_{3}\left(\mathrm{CH}_{2}\right) 6 \mathrm{CH}_{2} \mathrm{CH}_{2} \mathrm{CO}-\right\}, 0.86\{9 \mathrm{H}, \mathrm{m}$, $\left.3 \times \mathrm{CH}_{3}\left(\mathrm{CH}_{2}\right)_{8} \mathrm{CO}-\right\}$.

\section{Methyl 6-0-cinnamoyl-2,3,4-tri-0-lauroyl- $\alpha$ - D-glucopyranoside 8}

Yield (105 mg, 87\%) as colourless crystalline solid, m.p. $49-52^{\circ} \mathrm{C}, \mathrm{R}_{\mathrm{f}} 0.53$ (EtOAc: $\mathrm{nC}_{6} \mathrm{H}_{14}, 1: 6$ ). Anal. calcd. for $\mathrm{C}_{52} \mathrm{H}_{86} \mathrm{O}_{10}: \mathrm{C}, 71.72 ; \mathrm{H}, 9.89 \%$. Found: $\mathrm{C}, 71.79 ; \mathrm{H}, 9.94 \%$. FTIR 1716 (-CO), 1627 $(-\mathrm{CH}=\mathrm{CH}-) \mathrm{cm}^{-1} .{ }^{1} \mathrm{H}$ NMR $\left(\mathrm{CDCl}_{3}, \mathrm{ppm}\right): \delta_{\mathrm{H}}=7.71$ $(1 \mathrm{H}, \mathrm{d}, J=16.0 \mathrm{~Hz}, \mathrm{PhCH}=\mathrm{CHCO}), 7.50(2 \mathrm{H}, \mathrm{m}$, Ar-H), $7.35(3 \mathrm{H}, \mathrm{m}, \mathrm{Ar}-\mathrm{H}), 6.45(1 \mathrm{H}, \mathrm{d}, J=16.0 \mathrm{~Hz}$, $\mathrm{PhCH}=\mathrm{CHCO}-), 5.45(1 \mathrm{H}, \mathrm{d}, J=3.6 \mathrm{~Hz}, \mathrm{H}-1), 5.0$ $(1 \mathrm{H}, \mathrm{dd}, J=3.6$ and $10.1 \mathrm{~Hz}, \mathrm{H}-2), 4.89(1 \mathrm{H}, \mathrm{t}, J=$ $9.7 \mathrm{~Hz}, \mathrm{H}-3), 4.85(1 \mathrm{H}, \mathrm{t}, J=9.7 \mathrm{~Hz}, \mathrm{H}-4), 4.33(1 \mathrm{H}$, $\mathrm{dd}, J=2.0$ and $12.1 \mathrm{~Hz}, \mathrm{H}-6 \mathrm{~b}), 4.0(1 \mathrm{H}, \mathrm{dd}, J=4.8$ and $10.2 \mathrm{~Hz}, \mathrm{H}-6 \mathrm{a}), 3.95(1 \mathrm{H}, \mathrm{m}, \mathrm{H}-5), 3.35(3 \mathrm{H}$, $\mathrm{s}, 1-\mathrm{OCH} 3), 2.34\left\{6 \mathrm{H}, \mathrm{m}, 3 \times \mathrm{CH}_{3}\left(\mathrm{CH}_{2}\right) 9 \mathrm{CH}_{2} \mathrm{CO}-\right\}$, $1.64\left\{6 \mathrm{H}, \quad \mathrm{m}, \quad 3 \times \mathrm{CH}_{3}\left(\mathrm{CH}_{2}\right) 8 \mathrm{CH}_{2} \mathrm{CH}_{2} \mathrm{CO}-\right\}, 1.24$ $\left\{48 \mathrm{H}, \mathrm{m}, 3 \times \mathrm{CH}_{3}\left(\mathrm{CH}_{2}\right) 8 \mathrm{CH}_{2} \mathrm{CH}_{2} \mathrm{CO}\right\}, 0.85\{9 \mathrm{H}, \mathrm{m}$, $\left.3 \times \mathrm{CH}_{3}\left(\mathrm{CH}_{2}\right) 10 \mathrm{CO}-\right\}$.

\section{Methyl 6-0-cinnamoyl-2,3,4-tri-0-myristoyl- $\alpha$-D-glucopyranoside 9}

Yield (109 mg, 87\%) as colourless needles, m.p. $39-41^{\circ} \mathrm{C}, \mathrm{R}_{\mathrm{f}} 0.52$ (EtOAc: $\mathrm{nC}_{6} \mathrm{H}_{14}, 1: 5$ ). Anal. calcd. for $\mathrm{C}_{58} \mathrm{H}_{98} \mathrm{O}_{10}: \mathrm{C}, 72.95 ; \mathrm{H}, 10.27 \%$. Found: C, 72.99; H, 10.33\%. FTIR $1682(-\mathrm{CO}), 1635$ $(-\mathrm{CH}=\mathrm{CH}-) \mathrm{cm}^{-1} .{ }^{1} \mathrm{H}$ NMR $\left(\mathrm{CDCl}_{3}, \mathrm{ppm}\right): \delta_{\mathrm{H}}=7.70$ $(1 \mathrm{H}, \mathrm{d}, \mathrm{J}=16.1 \mathrm{~Hz}, \mathrm{PhCH}=\mathrm{CHCO}-), 7.53(2 \mathrm{H}, \mathrm{m}$, Ar-H) $7.38(3 \mathrm{H}, \mathrm{m}, \mathrm{Ar}-\mathrm{H}), 6.44(1 \mathrm{H}, \mathrm{d}, J=16.1$ $\mathrm{Hz}, \mathrm{PhCH}=\mathrm{CHCO}-), 5.50(1 \mathrm{H}, \mathrm{d}, J=3.6 \mathrm{~Hz}, \mathrm{H}-1)$, $5.13(1 \mathrm{H}, \mathrm{dd}, J=3.5$ and $9.8 \mathrm{~Hz}, \mathrm{H}-2), 4.98(1 \mathrm{H}, \mathrm{t}$, $J=9.6 \mathrm{~Hz}, \mathrm{H}-3), 4.83(1 \mathrm{H}, \mathrm{m}, \mathrm{H}-4), 4.36(1 \mathrm{H}, \mathrm{m}$, $\mathrm{H}-6 \mathrm{a}), 4.27(1 \mathrm{H}, \mathrm{m}, \mathrm{H}-6 \mathrm{~b}), 3.97(1 \mathrm{H}, \mathrm{m}, \mathrm{H}-5), 3.31$ $\left(3 \mathrm{H}, \mathrm{s}, 1-\mathrm{OCH}_{3}\right), 2.28\left\{6 \mathrm{H}, \mathrm{m}, 3 \times \mathrm{CH}_{3}\left(\mathrm{CH}_{2}\right)_{11} \mathrm{CH}_{2} \mathrm{CO}-\right\}$, $1.24\left\{66 \mathrm{H}, \mathrm{m}, 3 \times \mathrm{CH}_{3}\left(\mathrm{CH}_{2}\right)_{11} \mathrm{CH}_{2} \mathrm{CO}\right\}, 0.88\{9 \mathrm{H}, \mathrm{m}$, $\left.3 \times \mathrm{CH}_{3}\left(\mathrm{CH}_{2}\right)_{12} \mathrm{CO}-\right\}$.

\section{Methyl 6-0-cinnamoyl-2,3,4-tri-0-palmitoyl- $\alpha$-D-glucopyranoside 10}

Yield (109 mg, 95\%) as colourless needles, m.p. $54-56^{\circ} \mathrm{C}$, Rf 0.55 (EtOAc: $\mathrm{nC}_{6} \mathrm{H}_{14}, 1: 5$ ). Anal. Calcd. for $\mathrm{C}_{64} \mathrm{H}_{11} \mathrm{O}_{10}: \mathrm{C}, 73.99 ; \mathrm{H}, 10.60 \%$. Found: $\mathrm{C}, 74.06$; $\mathrm{H}, 10.67 \%$. FTIR $1740(-\mathrm{CO}), 1636\left(-\mathrm{CH}=\mathrm{CH}^{-}\right) \mathrm{cm}^{-1}$. ${ }^{1} \mathrm{H} \mathrm{NMR}\left(\mathrm{CDCl}_{3}, \mathrm{ppm}\right): \delta_{\mathrm{H}}=7.72(1 \mathrm{H}, \mathrm{d}, J=16.0 \mathrm{~Hz}$, $\mathrm{PhCH}=\mathrm{CHCO}-), 7.51(2 \mathrm{H}, \mathrm{m}, \mathrm{Ar}-\mathrm{H}), 7.36(3 \mathrm{H}, \mathrm{m}, \mathrm{Ar}-$ $\mathrm{H}), 6.46(1 \mathrm{H}, \mathrm{d}, J=16.0 \mathrm{~Hz}, \mathrm{PhCH}=\mathrm{CHCO}-), 5.51(1 \mathrm{H}$, 
$\mathrm{d}, J=3.7 \mathrm{~Hz}, \mathrm{H}-1), 5.33(1 \mathrm{H}, \mathrm{dd}, J=3.6$ and $10.2 \mathrm{~Hz}$, $\mathrm{H}-2), 5.13(1 \mathrm{H}, \mathrm{t}, J=9.6 \mathrm{~Hz}, \mathrm{H}-3), 4.95(1 \mathrm{H}, \mathrm{t}, J=9.7$ $\mathrm{Hz}, \mathrm{H}-4), 4.35(1 \mathrm{H}, \mathrm{dd}, J=4.8$ and $10.2 \mathrm{~Hz}, \mathrm{H}-6 \mathrm{a})$, $4.05(1 \mathrm{H}, \mathrm{t}, J=10.2 \mathrm{~Hz}, \mathrm{H}-6 \mathrm{~b}), 3.88(1 \mathrm{H}, \mathrm{m}, \mathrm{H}-5), 3.31$ $\left(3 \mathrm{H}, \mathrm{s}, 1-\mathrm{OCH}_{3}\right), 2.32\left\{6 \mathrm{H}, \mathrm{m}, 3 \times \mathrm{CH}_{3}\left(\mathrm{CH}_{2}\right)_{13} \mathrm{CH}_{2} \mathrm{CO}-\right\}$, $1.62\left\{6 \mathrm{H}, \quad \mathrm{m}, \quad 3 \times \mathrm{CH}_{3}\left(\mathrm{CH}_{2}\right)_{12} \mathrm{CH}_{2} \mathrm{CH}_{2} \mathrm{CO}-\right\}, 1.24$ $\left\{72 \mathrm{H}, \mathrm{m}, 3 \times \mathrm{CH}_{3}\left(\mathrm{CH}_{2}\right) 12\left(\mathrm{CH}_{2}\right)_{2} \mathrm{CO}\right\}, 0.86\{9 \mathrm{H}, \mathrm{m}$, $\left.3 \times \mathrm{CH}_{3}\left(\mathrm{CH}_{2}\right)_{14} \mathrm{CO}-\right\}$.

\section{Methyl 6-0-cinnamoyl-2,3,4-tri-0-pivaloyl- $\alpha$ - D-glucopyranoside 11}

Yield (83 mg, 69\%) as colourless pasty mass, $\mathrm{R}_{\mathrm{f}} 0.53$ (EtOAc: $\mathrm{nC}_{6} \mathrm{H}_{14}, 1: 6$ ). Anal. calcd. for C31H44O10: C, 64.58; H, 7.64\%. Found: C, 64.62; $\mathrm{H}, 7.68 \%$. FTIR $1724(-\mathrm{CO}), 1632(-\mathrm{CH}=\mathrm{CH}-) \mathrm{cm}$ 1. ${ }^{1} \mathrm{H}$ NMR $\left(\mathrm{CDCl}_{3}, \mathrm{ppm}\right): \delta \mathrm{H}=7.69(1 \mathrm{H}, \mathrm{d}, J=16.0$ $\mathrm{Hz}, \mathrm{PhCH}=\mathrm{CHCO}-), 7.47(2 \mathrm{H}, \mathrm{m}, \mathrm{Ar}-\mathrm{H}), 7.33(3 \mathrm{H}$, $\mathrm{m}, \mathrm{Ar}-\mathrm{H}), 6.46(1 \mathrm{H}, \mathrm{d}, \mathrm{J}=16.0 \mathrm{~Hz}, \mathrm{PhCH}=\mathrm{CHCO}-)$, $5.50(1 \mathrm{H}, \mathrm{d}, J=3.6 \mathrm{~Hz}, \mathrm{H}-1), 5.05(1 \mathrm{H}, \mathrm{dd}, J=3.7$ and $10.2 \mathrm{~Hz}, \mathrm{H}-2), 4.92(1 \mathrm{H}, \mathrm{t}, J=9.7 \mathrm{~Hz}, \mathrm{H}-3), 4.85$ $(1 \mathrm{H}, \mathrm{t}, J=9.6 \mathrm{~Hz}, \mathrm{H}-4), 4.30(1 \mathrm{H}, \mathrm{m}, \mathrm{H}-5), 4.14(2 \mathrm{H}$, $\mathrm{m}, \mathrm{H}-6 \mathrm{a}$ and $\mathrm{H}-6 \mathrm{~b}), 3.37\left(3 \mathrm{H}, \mathrm{s}, 1-\mathrm{OCH}_{3}\right), 2.34\{2 \mathrm{H}$ $\left.\mathrm{m}, \mathrm{CH}_{3}\left(\mathrm{CH}_{2}\right)_{11} \mathrm{CH}_{2} \mathrm{CO}-\right\}, 1.23\left\{27 \mathrm{H}, \mathrm{s}, 3 \times\left(\mathrm{CH}_{3}\right)_{3} \mathrm{CCO}-\right\}$, $1.17\left\{22 \mathrm{H}, \mathrm{m}, \mathrm{CH}_{3}\left(\mathrm{CH}_{2}\right)_{11} \mathrm{CH}_{2} \mathrm{CO}-\right\}, 0.85\{3 \mathrm{H}, \mathrm{m}$, $\left.\mathrm{CH}_{3}\left(\mathrm{CH}_{2}\right)_{12} \mathrm{CO}-\right\}$.

\section{Methyl 6-0-cinnamoyl-2,3,4-tri-0- methanesulfonyl- $\alpha$-D-glucopyranoside 12}

Yield ( $81 \mathrm{mg}, 81 \%$ ) as white homogenous syrup, $R_{f}$ 0.52 (EtOAc: $\mathrm{nC}_{6} \mathrm{H}_{14}, 1: 5$ ). Anal. calcd for $\mathrm{C}_{19} \mathrm{H}_{26} \mathrm{~S}_{3} \mathrm{O}_{13}$ : C, 40.86; $H, 4.66 \%$. Found: $C, 40.91 ; H, 4.70 \%$. FTIR $1740(-\mathrm{CO}), 1637(-\mathrm{CH}=\mathrm{CH}-), 1365\left(-\mathrm{SO}_{2}\right) \mathrm{cm}$ 1. ${ }^{1} \mathrm{H}$ NMR $\left(\mathrm{CDCl}_{3}, \mathrm{ppm}\right): \delta_{\mathrm{H}}=7.76(1 \mathrm{H}, \mathrm{d}, \mathrm{J}=16.2$ $\mathrm{Hz}, \mathrm{PhCH}=\mathrm{CHCO}-), 7.53(2 \mathrm{H}, \mathrm{m}, \mathrm{Ar}-\mathrm{H}), 7.38(3 \mathrm{H}, \mathrm{m}$, Ar-H), $6.45(1 \mathrm{H}, \mathrm{d}, J=16.1 \mathrm{~Hz}, \mathrm{PhCH}=\mathrm{CHCO}-), 5.12$ $(1 \mathrm{H}, \mathrm{d}, J=3.6 \mathrm{~Hz}, \mathrm{H}-1), 5.09(1 \mathrm{H}, \mathrm{dd}, J=3.6$ and $10.1 \mathrm{~Hz}, \mathrm{H}-2), 4.88(1 \mathrm{H}, \mathrm{t}, J=9.6 \mathrm{~Hz}, \mathrm{H}-3), 4.66(1 \mathrm{H}$, $\mathrm{t}, J=9.6 \mathrm{~Hz}, \mathrm{H}-4), 4.59(1 \mathrm{H}, \mathrm{dd}, J=4.9$ and $12.0 \mathrm{~Hz}$, $\mathrm{H}-6 \mathrm{a}), 4.42(1 \mathrm{H}, \mathrm{dd}, J=2.0$ and $12.1 \mathrm{~Hz}, \mathrm{H}-6 \mathrm{~b}), 4.08$ $(1 \mathrm{H}, \mathrm{ddd}, J=2.8,9.7$ and $12.6 \mathrm{~Hz}, \mathrm{H}-5), 3.47(3 \mathrm{H}$, $\left.\mathrm{S}, 1-\mathrm{OCH}_{3}\right), 3.19,3.15,3.08\left(3 \times 3 \mathrm{H}, 3 \times \mathrm{s}, 3 \times \mathrm{CH}_{3} \mathrm{SO}_{2}-\right)$.

\section{Methyl 2,3,4-tri-0-benzenesulfonyl-6-0- cinnamoyl- $\alpha$-D-glucopyranoside 13}

Yield (82 mg, 80\%) as colourless pasty mass, $\mathrm{R}_{\mathrm{f}} 0.52$ (EtOAc: $\mathrm{nC}_{6} \mathrm{H}_{14}, 1: 4$ ). Anal. calcd. for $\mathrm{C}_{35} \mathrm{H}_{32} \mathrm{~S}_{3} \mathrm{O}_{13}: \mathrm{C}, 55.56 ; \mathrm{H}, 4.23 \%$. Found: $\mathrm{C}, 55.61$; $\mathrm{H}, 4.25 \%$. FTIR $1712(-\mathrm{CO}), 1632(-\mathrm{CH}=\mathrm{CH}-), 1365$ $\mathrm{cm}^{-1}\left(-\mathrm{SO}_{2}\right) \mathrm{cm}^{-1} .{ }^{1} \mathrm{H}$ NMR $\left(\mathrm{CDCl}_{3}, \mathrm{ppm}\right): \delta \mathrm{H}=7.95$ $(6 \mathrm{H}, \mathrm{m}, \mathrm{Ar}-\mathrm{H}), 7.67(1 \mathrm{H}, \mathrm{d}, \mathrm{J}=16.1 \mathrm{~Hz}, \mathrm{PhCH}=\mathrm{CHCO}-)$
$7.64(3 \mathrm{H}, \mathrm{m}, \mathrm{Ar}-\mathrm{H}), 7.62(6 \mathrm{H}, \mathrm{m}, \mathrm{Ar}-\mathrm{H}), 7.53(2 \mathrm{H}, \mathrm{m}$, Ar-H), $7.38(3 \mathrm{H}, \mathrm{m}, \mathrm{Ar}-\mathrm{H}), 6.44(1 \mathrm{H}, \mathrm{d}, J=16.0 \mathrm{~Hz}$, $\mathrm{PhCH}=\mathrm{CHCO}-), 4.89(1 \mathrm{H}, \mathrm{d}, J=3.6 \mathrm{~Hz}, \mathrm{H}-1), 4.83$ $(1 \mathrm{H}, \mathrm{dd}, J=3.7$ and $10.1 \mathrm{~Hz}, \mathrm{H}-2), 4.75(1 \mathrm{H}, \mathrm{t}, J=$ $9.6 \mathrm{~Hz}, \mathrm{H}-3), 4.67(1 \mathrm{H}, \mathrm{m}, \mathrm{H}-4), 4.16(1 \mathrm{H}, \mathrm{m}, \mathrm{H}-6 \mathrm{a})$, $3.93(1 \mathrm{H}, \mathrm{m}, \mathrm{H}-6 \mathrm{~b}), 3.66(1 \mathrm{H}, \mathrm{m}, \mathrm{H}-5), 3.35(3 \mathrm{H}, \mathrm{s}$, $\left.1-\mathrm{OCH}_{3}\right)$.

\section{Methyl 2,3,4-tri-0-(4-chlorobenzoyl)-6-0- cinnamoyl- $\alpha$-D-glucopyranoside 14}

Yield (57 mg, 80\%) as colourless needles, m.p. 41$43^{\circ} \mathrm{C}, \mathrm{R}_{\mathrm{f}} 0.51$ (EtOAc: $\mathrm{nC}_{6} \mathrm{H}_{14}, 1: 5$ ). Anal. calcd. for $\mathrm{C}_{37} \mathrm{H}_{20} \mathrm{Cl}_{3} \mathrm{O}_{10}: \mathrm{C}, 60.78 ; \mathrm{H}, 2.74 \%$. Found: $\mathrm{C}, 60.87$; $\mathrm{H}, 2.80 \%$. FTIR $1730(-\mathrm{CO}), 1639 \mathrm{~cm}^{-1}(-\mathrm{CH}=\mathrm{CH}-)$ $\mathrm{cm}^{-1} .{ }^{1} \mathrm{H}$ NMR $\left(\mathrm{CDCl}_{3^{\prime}}, \mathrm{ppm}\right): \delta_{\mathrm{H}}=7.95(6 \mathrm{H}, \mathrm{m}, \mathrm{Ar}-\mathrm{H})$, $7.89(1 \mathrm{H}, \mathrm{d}, J=16.0 \mathrm{~Hz}, \mathrm{PhCH}=\mathrm{CHCO}-), 7.37(2 \mathrm{H}, \mathrm{m}$, Ar-H), $7.33(3 \mathrm{H}, \mathrm{m}, \mathrm{Ar}-\mathrm{H}), 6.48(1 \mathrm{H}, \mathrm{d}, J=16.0 \mathrm{~Hz}$, $\mathrm{PhCH}=\mathrm{CHCO}-), 5.60(1 \mathrm{H}, \mathrm{d}, J=3.6 \mathrm{~Hz}, \mathrm{H}-1), 5.27$ $(1 \mathrm{H}, \mathrm{dd}, J=3.6$ and $10.1 \mathrm{~Hz}, \mathrm{H}-2), 5.20(1 \mathrm{H}, \mathrm{t}, J=9.7$ $\mathrm{Hz}, \mathrm{H}-3), 5.12(1 \mathrm{H}, \mathrm{t}, J=9.6 \mathrm{~Hz}, \mathrm{H}-4), 4.72(1 \mathrm{H}, \mathrm{m}$, $\mathrm{H}-6 \mathrm{a}), 4.44(1 \mathrm{H}, \mathrm{t}, J=10.2 \mathrm{~Hz}, \mathrm{H}-6 \mathrm{~b}), 4.21(1 \mathrm{H}, \mathrm{m}$, $\mathrm{H}-5), 3.39\left(3 \mathrm{H}, \mathrm{S}, 1-\mathrm{OCH}_{3}\right)$.

\section{Antimicrobial screening studies Test microorganisms}

The synthesized test compounds (Scheme 1) were subjected to antibacterial and antifungal screening against Gram-positive \& Gram-negative bacterial strains and phytopathogenic fungi (Table 2). In all cases, a $2 \%$ solution (in $\mathrm{CHCl} 3$ ) of the chemicals was used. Test tube cultures of bacterial and fungal pathogens were obtained from the Microbiology Laboratory, Department of Microbiology, University of Chittagong, Bangladesh.

\section{Screening of antibacterial activity}

The in vitro antibacterial spectrum of the synthesized chemicals were done by disc diffusion method [37] with little modification [38]. Sterilized paper discs of $4 \mathrm{~mm}$ in diameter and Petri dishes of $150 \mathrm{~mm}$ in diameter were used throughout the experiment. The autoclaved Mueller-Hinton agar medium, cooled to $45^{\circ} \mathrm{C}$, was poured into sterilized Petri dishes to a depth of 3 to $4 \mathrm{~mm}$ and after solidification of the agar medium the plates were transferred to an incubator at $37^{\circ} \mathrm{C}$ for 15 to 20 minutes to dry off the moisture that developed on the agar surface. The plates were inoculated with the standard bacterial suspensions (as 
Table 2. List of tested bacterial and fungal microorganisms.

\begin{tabular}{ccc}
\hline Types of bacteria/fungi & Name of tested bacteria/fungi & Strain no. \\
\hline Gram +Ve & Bacillus cereus & BTCC 17 \\
\cline { 2 - 3 } Gram -Ve & Staphylococcus aureus & ATCC 25923 \\
\hline Fungi & Escherichia coli & ATCC 25922 \\
\hline & Pseudomonas aeruginosa & CRL (ICDDR'B) \\
\hline
\end{tabular}

McFarland 0.5 standard) followed by spread plate method and allowed to dry for three to five minutes. Dried and sterilized filter paper discs were treated separately with $50 \mu \mathrm{g}$ dry weight/ disc from $2 \%$ solution (in $\mathrm{CHCl}_{3}$ ) of each test chemical using a micropipette, dried in air under aseptic condition and were placed at equidistance in a circle on the seeded plate. A control plate was also maintained in each case without any test chemical. These plates were kept for 4-6 hours at low temperature $\left(4-6^{\circ} \mathrm{C}\right)$ and the test chemicals diffused from disc to the surrounding medium. The plates were then incubated at $35 \pm 2^{\circ} \mathrm{C}$ for 24 hours to allow maximum growth of the microorganisms. The antibacterial activity of the test agent was determined by measuring the mean diameter of zone of inhibitions (in millimeter). Each experiment was repeated thrice. All the results were compared with the standard antibacterial antibiotic Ampicillin (20 $\mu \mathrm{g} /$ disc, BEXIMCO Pharm. Bangladesh Ltd).

\section{Screening of mycelial growth assay}

The antifungal activities of the D-glucopyranoside derivatives (scheme-1) were investigated by food poisoned technique [39]. Two percent solution of the test chemical (in $\mathrm{CHCl}_{3}$ ) was mixed with sterilized melted Saburaud agar medium to obtain the desired concentration (2\%) and this was poured in sterilized Petri dishes. At the center of each plate, 5 days old fungal mycelial block (4 $\mathrm{mm}$ in diameter) was inoculated and incubated at $27^{\circ} \mathrm{C}$. A control set was also maintained in each experiment. Linear mycelial growth of fungus was measured after 3-5 days of incubation. The percentage inhibition of radial mycelial growth of the test fungus was calculated as follows: $I=[(C-T) / T] \times 100$
Where, $\mathrm{I}=$ Percentage of inhibition, $\mathrm{C}=$ Diameter of the fungal colony in control $\left(\mathrm{CHCl}_{3}\right), \mathrm{T}=$ Diameter of the fungal colony in treatment. All the results were compared with the standard antifungal antibiotic nystatin $(100 \mu \mathrm{g} / \mathrm{ml}$ medium, BEXIMCO Pharm. Bangladesh Ltd.).

\section{RESULTS AND DISCUSSION Chemistry and spectral characterization}

The main objective of the work reported here was to study regioselective cinnamoylation of methyl $\alpha$-D-glucopyranoside (1) using the direct acylation method (Scheme 1, Table 1). Although this method is not a unique one and gives mixture of products which require tedious separation procedures, we have to employ it due to constraints in facilities. Cinnamoylation of methyl $\alpha$-D-glucopyranoside (1) in pyridine at freezing temperature, followed by usual work-up procedure, afforded two compounds 2 and 3 . However, we were able to separate 2 (53\%) and $3(28 \%)$ by using conventional column chromatography method in a pure form. The resulting acylation product (2) was then converted to a series of derivatives using various acylating agents containing a wide variety of biologically prone atoms/groups (Table 1). The selection of various acylating agents was deliberate with the aim of finding biologically active agents.

Our initial effort was to carry out selective acylation of methyl $\alpha$-D-glucopyranoside (1) with unimolecular amount of non-traditional acylating agent cinnamoyl chloride in pyridine at $-5^{\circ} \mathrm{C}$. Conventional work-up procedure, followed by removal of solvent and silica gel column chromatographic separation, we were able to 
separate compound (2) in 53\% yield and also compound 3 in $28 \%$ yield. Both the compounds were obtained as chromatographically thick syrup.

The structure of the cinnamoyl derivative (2) was established by analyzing its IR and ${ }^{1} \mathrm{H}$-NMR spectra. The IR spectrum of this compound showed the following characteristic peaks: 1638 $\mathrm{cm}^{-1}(-\mathrm{CH}=\mathrm{CH}-), 1728(-\mathrm{CO})$ and $3385-3525 \mathrm{~cm}^{-1}(\mathrm{br}$ $-\mathrm{OH}$ stretching). The $1 \mathrm{H}-\mathrm{NMR}$ spectrum showed a two-proton multiplet at $\delta 7.47$ and a three-proton multiplet at $\delta 7.33$ due to the aromatic protons. Also, two characteristic one-proton doublets at $\delta$ $7.69(\mathrm{~J}=16.0 \mathrm{~Hz}, \mathrm{PhCH}=\mathrm{CHCO})$ and $\delta 6.46(\mathrm{~J}=16.0$ $\mathrm{Hz}, \mathrm{PhCH}=\mathrm{CHCO}-$ ) was due to the olefinic protons of one cinnamoyl group, thereby suggesting the introduction of one cinnamoyl group in the molecule. The downfield shift of C- 6 proton to 4.56 (as dd, $J=5.1$ and $12.2 \mathrm{~Hz}, \mathrm{H}-6 \mathrm{a}$ ) and 4.18 (as $\mathrm{dd}, J=2.1$ and $12.1 \mathrm{~Hz}, \mathrm{H}-6 \mathrm{~b}$ ) from their usual values in the precursor compound 1 and the resonances of other protons in their anticipated positions, showed the presence of the cinnamoyl group at position 6. By complete analysis of the IR and $1 \mathrm{H}$-NMR spectra, the structure of this compound was assigned as methyl 6-O-cinnamoyl- $\alpha-D$ glucopyranoside (2).

The IR spectrum of the higher-moving component (3), displayed the following absorption bands at 3360-3510 (br) (for $-\mathrm{OH}) 1735 \mathrm{~cm}^{-1}$ (for - $\mathrm{CO}$ stretching) and $1631 \mathrm{~cm}^{-1}$ (for $-\mathrm{CH}=\mathrm{CH}$ ) stretchings. In the ${ }^{1} \mathrm{H}-\mathrm{NMR}$ spectrum (Fig.-04) two one-proton doublets at $\delta 7.72,7.68(2 \times 1 \mathrm{H}, 2 \times \mathrm{d}, \mathrm{J}=$ $16.0 \mathrm{~Hz}, 2 \times \mathrm{PhCH}=\mathrm{CHCO}-)$ and also two one-proton doublets at $\delta 6.48,6.44(2 \times 1 \mathrm{H}, 2 \times \mathrm{d}, J=16.1 \mathrm{~Hz}$, $2 \times \mathrm{PhCH}=\mathrm{CHCO}-)$ due to the introduction of two cinnamoyl groups in the molecule. In addition a four-proton multiplet at $\delta 7.45$ (as $\mathrm{m}, \mathrm{Ar}-\mathrm{H}$ ) and a six-proton multiplet at $\delta 7.34$ (as, m, Ar-H) due to the two aromatic ring protons. Complete analysis of its IR and spectra of its derivatives led us to assign its structure as methyl 2,6-di-O-cinnamoyl$\alpha$-D-glucopyranoside (3).

Further support for the structure of the cinnamoyl derivative (2) was obtained by its conversion to and identification of the acetyl derivative (4). Thus, treatment of compound 2 with acetic anhydride in pyridine, followed by usual aqueous work-up and column chromatographic purification, provided the the tri-O-acetyl derivative (4) in $72 \%$ yield as semi solid. The IR spectrum of the compound showed the following absorption bands: 1712, $1778 \mathrm{~cm}^{-1}$ (-CO) and $1628 \mathrm{~cm}^{-1}(-\mathrm{CH}=\mathrm{CH}-)$. The introduction of three acetyl group in the molecule was attributed by the appearance of three three-proton singlet at 2.05, 2.01 and 1.98 in its ${ }^{1} \mathrm{H}$-NMR spectrum. The C-2 proton resonated at $\delta 5.46$ (as dd, $J=3.6$ and $10.1 \mathrm{~Hz}$ ) and shifted downfield from the precursor triol (2) ( $\delta 3.43$ ); C-3 proton resonated at $\delta 5.02$ (as $t, J=9.7 \mathrm{~Hz}$ ) and shifted downfield from the precursor triol (2) ( $\delta$ 3.79); also, C-4 proton resonated downfield to $\delta 4.99$ (as $t, J=9.7 \mathrm{~Hz}$ ) as compared to the precursor compound 2 ( $\delta 3.56$ ), thereby suggesting the attachment of the acetyl groups at positions 2, 3 and 4 . The resonances of other protons were observed in their anticipated positions. The structure of the acetate was thus assigned as methyl 2,3,4-tri-O-acetyl-6-0cinnamoyl- $\alpha$-D-glucopyranoside (4) by complete analysis of its IR and ${ }^{1} \mathrm{H}-\mathrm{NMR}$ spectra.

The structure of the cinnamoyl derivative (2) was then derivatised using a number of fatty acid chlorides in order to get further support to its structure and also prepare newer products. Thus, treatment of compound 2 with pentanoyl chloride in pyridine, followed by usual workup and chromatographic purification gave the pentanoyl derivative (5) in $88.8 \%$ yield as thick syrup. Its IR spectrum, the absorption bands at $1758 \mathrm{~cm}^{-1}(-\mathrm{CO})$ and $1630 \mathrm{~cm}^{-1}(-\mathrm{CH}=\mathrm{CH}-)$ stretching. In its ${ }^{1} \mathrm{H}-\mathrm{NMR}$ spectrum, three sixproton multiplet at $\delta 2.31\left\{3 \times \mathrm{CH}_{3}\left(\mathrm{CH}_{2}\right) 2 \mathrm{CH}_{2} \mathrm{CO}-\right\}$, $\delta \quad 1.61\left\{3 \times \mathrm{CH}_{3} \mathrm{CH}_{2} \mathrm{CH}_{2} \mathrm{CH}_{2} \mathrm{CO}-\right\}$ and $\delta 1.33$ $\left\{3 \times \mathrm{CH}_{3} \mathrm{CH}_{2}\left(\mathrm{CH}_{2}\right)_{2} \mathrm{CO}\right\}$ and one nine-proton multiplet at $\delta 0.88\left\{3 \times \mathrm{CH}_{3}\left(\mathrm{CH}_{2}\right)_{3} \mathrm{CO}\right\}$, indicated the presence of three pentanoyl groups in the molecule. The deshielding of C-2, C-3 and C-4 protons to $\delta 4.95$ (as dd, $J=3.6$ and $10.0 \mathrm{~Hz}$ ), $\delta$ 4.85 (as $\mathrm{t}, \mathrm{J}=9.6 \mathrm{~Hz}$ ) and $\delta 4.62$ (as $\mathrm{t}, \mathrm{J}=9.5 \mathrm{~Hz}$ ) from their precursor compound 2 values $(\delta 3.43$ ), ( $\delta 3.79$ ) and ( $\delta 3.56)$, respectively indicated the introduction of the three pentanoyl groups at positions 2, 3 and 4. Complete analysis of its IR and ${ }^{1} \mathrm{H}-\mathrm{NMR}$ spectrum enabled us to assign its structure as methyl 6-0-cinnamoyl-2,3,4-tri-Opentanoyl- $\alpha$-D-glucopyranoside (5).

Reaction of compound 2 with hexanoyl 
chloride in pyridine medium, followed by conventional aqueous work-up procedure and silica gel column chromatographic purification provided the hexanoyl derivative (6) in $90 \%$ yield as needles m.p. $48^{\circ} \mathrm{C}$. In its IR spectrum, the absorption bands at $1775 \mathrm{~cm}^{-1}$, and 1622 $\mathrm{cm}^{-1}$ corresponded to $-\mathrm{CO}$ and $(-\mathrm{CH}=\mathrm{CH}-)$ stretchings, respectively. The $1 \mathrm{H}-\mathrm{NMR}$ spectrum of compound 6 displayed two six-proton multiplet at $\delta 2.26\left\{3 \times \mathrm{CH}_{3}\left(\mathrm{CH}_{2}\right) 3 \mathrm{CH}_{2} \mathrm{CO}-\right\}$, and $\delta$ $1.60\left\{3 \times \mathrm{CH}_{3}\left(\mathrm{CH}_{2}\right) 2 \mathrm{CH}_{2} \mathrm{CH} 2 \mathrm{CO}-\right\}$, a twelve-proton multiplet at $\delta 1.24 \quad\left\{3 \times \mathrm{CH}_{3}\left(\mathrm{CH}_{2}\right) 2 \mathrm{CH}_{2} \mathrm{CH}_{2} \mathrm{CO}-\right\}$ and one nine-proton multiplet at $\delta 0.87$ $\left\{3 \times \mathrm{CH}_{3}\left(\mathrm{CH}_{2}\right) 4 \mathrm{CO}\right\}$ showing the attachment of three hexanoyl groups. The resonance for $\mathrm{C}-2$, C-3 and C-4 appeared at $\delta 5.12$ (as dd, $J=3.6$ and $10.1 \mathrm{~Hz}$ ), $\delta 4.95$ (as t, J=9.6 Hz) and $\delta 4.89$ (as $\mathrm{t}, J=9.7 \mathrm{~Hz}$ ) which shifted downfield from their values (compound 2 ) indicating the attachment of the hexanoyl groups at positions 2, 3 and 4 . The rest of the IR and $1 \mathrm{H}$-NMR spectra was in complete agreement with the structure assigned as methyl 6-0-cinnamoyl-2,3,4-tri-0-hexanoyl- $\alpha$ D-glucopyranoside (6).

Cinnamoyl derivative 2 was then converted to the decanoyl derivative (7) in $86.4 \%$ as homogeneous syrup. Its IR spectrum displayed the following absorption bands: $1688 \mathrm{~cm}^{-1}(-\mathrm{CO})$ and, $1642 \mathrm{~cm}^{-1}(-\mathrm{CH}=\mathrm{CH}-)$. In its ${ }^{1} \mathrm{H}$-NMR spectrum, two six-proton multiplet at $\delta \quad 2.34 \quad\left\{3 \times \mathrm{CH}_{3}\left(\mathrm{CH}_{2}\right) 7 \mathrm{CH}_{2} \mathrm{CO}-\right\}$ and 1.62 $\left\{3 \times \mathrm{CH}_{3}\left(\mathrm{CH}_{2}\right)_{6} \mathrm{CH}_{2} \mathrm{CH}_{2} \mathrm{CO}-\right\}$, a thirty six-proton multiplet at $\delta 1.23\left\{3 \times \mathrm{CH}_{3}\left(\mathrm{CH}_{2}\right)_{6} \mathrm{CH}_{2} \mathrm{CH}_{2} \mathrm{CO}\right\}$ and a nine-proton multiplet at $\delta 0.86\left\{3 \times \mathrm{CH}_{3}\left(\mathrm{CH}_{2}\right)_{8} \mathrm{CO}-\right\}$ due to the presence of three decanoyl groups to the molecule. The downfield shift of $\mathrm{C}-2, \mathrm{C}-3$ and $\mathrm{C}-4$ protons to $\delta 5.07, \delta 4.85$ and $\delta 4.77$ from their usual values, indicative the attachment of the three decanoyl groups at positions 2, 3 and 4. On the basis of complete analysis of the IR and ${ }^{1} \mathrm{H}-\mathrm{NMR}$ spectra of this compound was accorded as methyl 6-0-cinnamoyl-2,3,4-tri-O-decanoyl- $\alpha$ D-glucopyranoside (7).

The cinnamoyl derivative (2) was then subjected to lauroylation, after usual work-up and separation by silica gel column chromatography furnished the lauroyl derivative (8) in $87.5 \%$ yield as needless m.p. $49-52^{\circ} \mathrm{C}$. The IR spectrum of this compound (8) displayed absorption bands at 1716 $\mathrm{cm}^{-1}$ and $1627 \mathrm{~cm}^{-1}$ due to carbonyl and olefinic stretchings. Its ${ }^{1} \mathrm{H}-\mathrm{NMR}$ spectrum displayed two sixproton multiplet at $2.34\left\{3 \times \mathrm{CH}_{3}\left(\mathrm{CH}_{2}\right)_{9} \mathrm{CH}_{2} \mathrm{CO}\right\}, \delta$ $1.64\left\{3 \times \mathrm{CH}_{3}\left(\mathrm{CH}_{2}\right)_{8} \mathrm{CH}_{2} \mathrm{CH}_{2} \mathrm{CO}-\right\}$ a forty eight-proton multiplet at $\delta 1.24\left\{3 \times \mathrm{CH}_{3}\left(\mathrm{CH}_{2}\right) 8 \mathrm{CH}_{2} \mathrm{CH}_{2} \mathrm{CO}\right\}$ and a nine-proton multiplet at $\delta 0.85\left\{3 \times \mathrm{CH}_{3}\left(\mathrm{CH}_{2}\right)_{10} \mathrm{CO}-\right\}$, thereby suggesting the presence of three lauroyl groups in the compound. Also the $\mathrm{C}-2, \mathrm{C}-3$ and $\mathrm{C}-4$ protons were deshielded considerably to $\delta 5.0$ (as dd, $J=3.6$ and $10.1 \mathrm{~Hz}$ ), $\delta 4.89$ (as t, $J=$ $9.7 \mathrm{~Hz}$ ) and $\delta 4.85$ (as $\mathrm{t}, J=9.7 \mathrm{~Hz}$ ) from their usual values, thus showing that the three lauroyl groups were introduced at positions 2, 3 and 4. So, analysis of rest of the IR and $1 \mathrm{H}-\mathrm{NMR}$ spectra, the structure of the lauroyl derivative was assigned as methyl 6-O-cinnamoyl-2,3,4-tri-O-lauryl- $\alpha$-Dglucopyranoside (8).

The triol (2) was then allowed to react with myristoyl chloride in dry pyridine and after usual work-up and chromatographic purification, we obtained compound 9 in $87.2 \%$ yield as needless, m.p. $39-40^{\circ} \mathrm{C}$. Its IR spectrum showed absorption bands at $1682 \mathrm{~cm}^{-1}$ (-CO stretching) and $1635 \mathrm{~cm}^{-1}(-\mathrm{CH}=\mathrm{CH}$ - stretching). In its $1 \mathrm{H}-\mathrm{NMR}$ spectrum, a six-proton multiplet at $\delta 2.28$ $\left\{3 \times \mathrm{CH}_{3}\left(\mathrm{CH}_{2}\right)_{11} \mathrm{CH}_{2} \mathrm{CO}-\right\}$, a sixty-six proton multiplet at $\delta 1.24\left\{3 \times \mathrm{CH}_{3}\left(\mathrm{CH}_{2}\right)_{11} \mathrm{CH}_{2} \mathrm{CO}-\right\}$ and a nine-proton multiplet at $\delta 0.88\left\{3 \times \mathrm{CH}_{3}\left(\mathrm{CH}_{2}\right)_{12} \mathrm{CO}-\right\}$ indicated the attachment of three myristoyl groups in the molecule. The downfield shift of the $\mathrm{C}-2, \mathrm{C}-3$ and $\mathrm{C}-4$ protons to $\delta 5.13, \delta 4.98$ and $\delta 4.83$ from their values and the resonances of other protons in their anticipated positions showed the attachment of myristoyl groups at positions 2, 3 and 4. Analysis of the IR and ${ }^{1} \mathrm{H}$-NMR spectra of this compound was in agreement with the structure established as methyl 6-0-cinnamoyl-2,3,4-tri-0-myristoyl- $\alpha$ D-glucopyranoside (9).

Further support for the structure accorded to compound 2, we used palmitoyl chloride for derivatizing compound 2 by direct acylation method. After usual work-up and purification procedure, we obtained the palmitoyl derivative (10) in $93.16 \%$ yield as needless, m.p. $54-56^{\circ} \mathrm{C}$. By complete analysis of its $I R$ and $1 \mathrm{H}-\mathrm{NMR}$ spectrum and by analogy with similar derivatives described earlier, the structure of this compound was confidently assigned as methyl 6-0-cinnamoyl-2,3,4-tri-0-palmitoyl- $\alpha$-D- 
glucopyranoside (10).

Our next effort was to treat compound 2 with pivaloyl chloride in dry pyridine and the corresponding pivaloyl derivative (11) in $69.16 \%$ yield as pasty mass. The IR spectrum of compound 11 showed peaks at $1724 \mathrm{~cm}^{-1}$ and $1632 \mathrm{~cm}^{-1}$ due to carbonyl and olefinic stretchings, respectively. In the ${ }^{1} \mathrm{H}-\mathrm{NMR}$ spectrum of 11 a twenty seven-proton singlet at $\delta 1.23\{3 \times(\mathrm{CH} 3) 3 \mathrm{CCO}\}$ was due to the methyl protons of pivaloyl groups which indicated the introduction of three pivaloyl groups. The downfield shift of $\mathrm{C}-2$ proton to $\delta 5.05$ (as dd, $\mathrm{J}=3.7$ and $10.2 \mathrm{~Hz}$ ), $\mathrm{C}-3$ proton to $\delta 4.92(\mathrm{t}, \mathrm{J}=9.7$ $\mathrm{Hz}$ ) and $\mathrm{C}-4$ proton to $\delta 4.85$ (as $\mathrm{t}, \mathrm{J}=9.6 \mathrm{~Hz}$ ) from their precursor triol $\delta$ values (2) showed the attachment of the pivaloyl groups at positions 2 3 and 4 . Complete analysis of the IR and ${ }^{~} \mathrm{H}-\mathrm{NMR}$ spectra was consistent with the structure of the compound assigned as methyl 6-0-cinnamoyl2,3,4-tri-O-pivaloyl- $\alpha$-D-glucopyranoside (11).

The structure of compound 2 was also supported by its transformation to and identification of the methanesulphonyl derivative (12). Compound 12 was prepared in $81 \%$ yield as clear syrup by using methanesulphonyl chloride in pyridine at freezing temperature. Its IR spectrum exhibited absorption bands at 1740, 1637 and 1365 $\mathrm{cm}-1$ due to $-\mathrm{CO}$ stretching, $-\mathrm{CH}=\mathrm{CH}-$ stretching and $-\mathrm{SO}_{2}$ stretching respectively. The presence of three methanesulphonyl groups in the molecule was demonstrated by its $1 \mathrm{H}$-NMR spectrum which displayed three three-proton singlets at $\delta 3.19, \delta$ 3.15 and $\delta 3.08$ due to the methyl protons of three methanesulphonyloxy $\left(3 \times \mathrm{CH}_{3} \mathrm{SO}_{2}^{-}\right)$groups. Also, the $\mathrm{C}-2, \mathrm{C}-3$ and $\mathrm{C}-4$ protons shifted downfield from its precursor compound 2 , thereby suggesting the attachment of the methanesulphonyl groups at positions 2, 3 and 4. Complete analysis of the $\mathrm{IR}$ and ${ }^{1} \mathrm{H}-\mathrm{NMR}$ spectra led us to establish its structure as methyl 6-0-cinnamoyl-2,3,4-tri-0methanesulfonyl- $\alpha$-D-glucopyranoside (12).

Benzenesulfonylation of triol (2) in pyridine followed by usual work up and separation by silica gel column chromatography, provided compound 13 in $80.39 \%$ yield as pasty mass. IR spectrum of this compound displayed absorption bands at 1712 $\mathrm{cm}^{-1}$ (-CO stretching), $1365 \mathrm{~cm}^{-1}$ (- $\mathrm{SO}_{2}$ stretching) and $1632 \mathrm{~cm}^{-1}\left(-\mathrm{CH}=\mathrm{CH}\right.$ - stretching). In its ${ }^{1} \mathrm{H}-\mathrm{NMR}$ spectrum, the peaks at $\delta 7.95(6 \mathrm{H}, \mathrm{m}), \delta 7.64(3 \mathrm{H}$, $\mathrm{m})$ and $\delta 7.62(6 \mathrm{H}, \mathrm{m})$ corresponded the protons of three phenyl groups. The downfield shift of $\mathrm{C}-2$ to $\delta 4.83$ (as dd, J=3.7 and $10.1 \mathrm{~Hz}$ ), C-3 to $\delta 4.75$ (as t, $J=9.6 \mathrm{~Hz}$ ) and $\mathrm{C}-4$ to $\delta 4.67$ (as m) from their precursor compound (2) ascertained the attachment of benzenesulfonyl groups at positions 2, 3 and 4. Complete analysis of the IR and $1 \mathrm{H}-\mathrm{NMR}$ spectra, led us to assign its structure as methyl 6-O-cinnamoyl-2,3,4-tri-Obenzenesulfonyl- $\alpha$-D-glucopyranoside (13).

Our final effort was to derivatised compound 2 using 4-chlorobenzoyl chloride by direct acylation method. Employing same work-up and purification techniques, we isolated compound (14) as needles, m.p. 41-430C. IR spectrum showed absorption bands at $1730 \mathrm{~cm}^{-1}$ (for - $\mathrm{CO}$ stretching) and $1639 \mathrm{~cm}^{-1}$ (for $-\mathrm{CH}=\mathrm{CH}^{-}$stretching). In its ${ }^{1} \mathrm{H}$-NMR spectrum, the two six-aromatic proton multiplet at $\delta 7.95$ (as m) and $\delta 7.74$ (as m) are characteristic of $\mathrm{p}$-substituted benzoyl groups. The deshielding of $\mathrm{C}-2, \mathrm{C}-3$ and $\mathrm{C}-4$ protons from their usual values and the resonance of other protons in their anticipated positions confirmed the structure of this compound as methyl 2,3,4-tri-0-(4-chlorobenzoyl)-6-0-cinnamoyl- $\alpha$ D-glucopyranoside (14).

Thus, regioselective cinnamoylation of methyl -D-glucopyranoside (1) by applying the direct method was unique in that all the reactions provided a single monosubstitution product in reasonably high yields. Though in case of the reation with cinnamoyl chloride, small amount of the disubstitution products ( 2 and 3 ) were formed but it successfully separated by column chromatography. The 6-0-cinnamoyl derivative (2) was further derivatized using a series of acyl chlorides. These acyl chlorides were chosen so as to contain probable biologically prone atoms/groups in order to find biologically active D-glucopyranoside derivatives. All the products thus prepared were employed as test chemicals for evaluating their antibacterial and antifungal activities against a number of human and plant pathogens. 
Table 3. Growth inhibition activity against Gram-positive and Gram-negative organisms by the glucopyranoside derivatives with standard antibiotic.

Growth inhibition zone $(\mathrm{mm})$ at $200 \mu \mathrm{g} \mathrm{dw/disc}$

\begin{tabular}{|c|c|c|c|c|}
\hline \multirow[t]{2}{*}{ Compound SI. } & \multicolumn{2}{|c|}{ Gram-positive organisms } & \multicolumn{2}{|c|}{ Gram-negative organisms } \\
\hline & B. subtilis & S. aureus & E. coli & P. aeruginosa \\
\hline 2 & NA & NA & NA & NA \\
\hline 3 & NA & NA & NA & NA \\
\hline 4 & 10 & 5 & 6 & 6.5 \\
\hline 5 & 10 & 6 & 6 & NA \\
\hline 6 & 5 & NA & NA & NA \\
\hline 7 & $* 16$ & $* 14$ & 10 & *16 \\
\hline 8 & $* 14$ & 7 & NA & $* 13$ \\
\hline 9 & 6 & 5.5 & NA & 5.5 \\
\hline 10 & 4.5 & NA & NA & NA \\
\hline 11 & NA & 4.5 & NA & NA \\
\hline 12 & NA & NA & NA & NA \\
\hline 13 & NA & NA & NA & 5 \\
\hline 14 & 5 & NA & NA & 6 \\
\hline **Ampicillin & $* 25$ & $* 21$ & $* 25$ & $* 17$ \\
\hline
\end{tabular}

Note: ${ }^{\prime * \prime}=$ good inhibition, ${ }^{\prime * * \prime}=$ standard antibiotic, 'dw' $=$ dry weight, 'NA' = not available

\section{Biological screening studies}

The results of the in vitro antibacterial screening against Gram-positive and Gram-negative human pathogens are shown in Table 3. Highest inhibition by acylated derivative 7 was observed against Gram-positive B. subtilis $(16 \mathrm{~mm})$ and Gramnegative $P$. aeruginosa (16 $\mathrm{mm}$ ). Test compounds $4,5,7,8$ and 9 were found to be active against all the Gram-positive bacteria, while 4, 7 and 8 were very active against Gram-negative bacteria tested herein (Table 3, Figure 1). Whereas, the test compounds 4 and 7 were recorded active against all the four Gram-positive and Gram-negative test bacteria. So, these compounds may be targeted for future studies for their usage as broad spectrum antibiotics. No inhibition was observed with the compounds 2, 3 and 12 against all the bacterial strains. The synthesized compounds showed a different degree of inhibitory activity against both Gram-positive and Gram-negative test bacteria.
In general, it has been observed that antibacterial results of the selectively acylated glucopyranoside derivatives obtained by using various acylating agents follow the order for Gram-positive organisms: $7>8>4=5>9>6=$ $14>10=11$ and Gram-negative bacteria follow the order: $7>8>4>5=14>9>13$. Another noteworthy observation was that the glucopyranoside derivatives were found comparatively more prone against Gram-positive organism than that of Gram-negative microorganisms. Grampositive bacteria are characterized by having as part of their cell wall structure peptidoglycan as well as polysaccharides. The hydrogen bonding between the cell wall and the hydrophilic moiety is therefore stronger than the Gram-negative bacteria. Having its polar head anchored in the membrane, the hydrophobic tail can interact with the lipid membrane, causing distortions leading to cell death. These results are in concurrence with the findings of our previous results $[26,31$, 33]. 
A

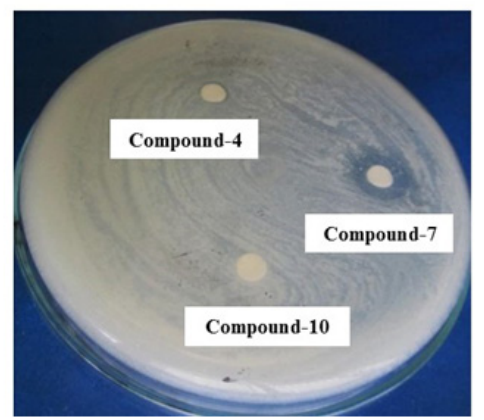

B

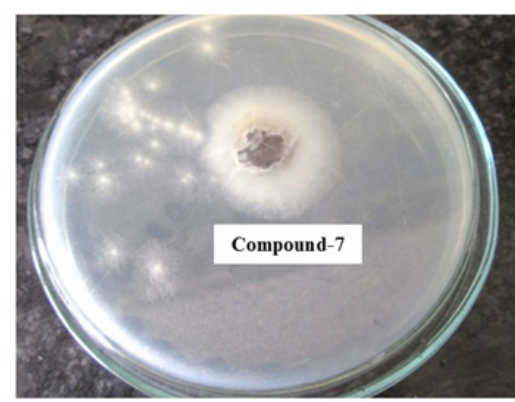

Figure 1. (A): Zone of inhibition observed against Staphylococcus aureus by three test chemicals 4,7 and 10. (B): Mycelial growth inhibition produced by test chemical 7 against Aspergillus niger.

A

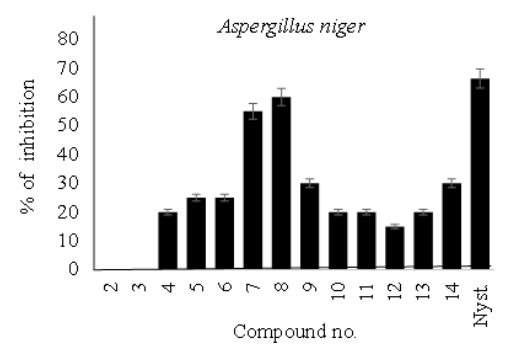

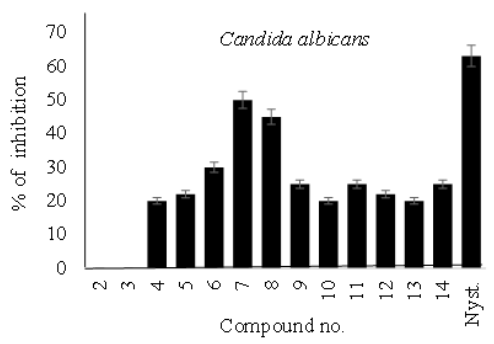

Figure 2. Percentage of inhibition of against (A) Aspergillus niger and (B) Candida albicans by the synthesized compounds (2-14) and standard antibiotic, Nystatin.

The percent inhibition of fungal mycelial growth by the synthesized test compounds and the standard antibiotic, Nystatin is furnished in Figure 2. The results revealed that the fungal strain A. niger was very sensitive towards the test compounds 7 (55.00\%) (Figure 1) and 8 (60.00\%) which were of comparable activities than that of standard antibiotic, Nystatin (66.40\%). The growth of $A$. niger was found to be inhibited by the test compounds $9(30.00 \%)$ and $14(30.00 \%)$ were also remarkable. On the other hand, compounds $6(30.00 \%), 7(50.00 \%)$ and 8 (45.00\%) showed good inhibition against Candida albicans though not as high as the standard antibiotic. Antifungal activity of our test compounds are in accordance with the results we observed before $[36,40]$. We also observed that most of the test compounds were active towards the mycelial growth of fungi in different level.
Thus, we observed that the high antibacterial and antifungal efficiency of the test compounds were due to the presence of different acyl groups like pentanoyl, decanoyl, lauroyl, myristoyl, 4-chlorobenzoyl etc. groups. These highly efficient antimicrobial agents can be chosen for the further work on the development of medicines for human disease control.

\section{CONCLUSIONS}

In conclusions, various D-glucopyranoside derivatives were prepared successfully with the objective of developing antimicrobial agents with excellent inhibition activity. Among these compounds, methyl 6-0-cinnamoyl-2,3,4tri-O-decanoyl- $\alpha$-D-glucopyranoside (7) and methyl 6-0-cinnamoyl-2,3,4-tri-0-lauroyl$\alpha$-D-glucopyranoside (8) showed the most prominent and potential activities against all of the microorganisms tested. Therefore, these 
compounds may represent a productive chemicals for the development of a new class of dual antibacterial and antifungal agents. This piece of work, in our opinion, has created an opportunity for further work with these test compounds, ultimately leading to establish new pesticides or medicines for human disease control with less environmental risks.

\section{ACKNOWLEDGEMENTS}

This research work was supported by the Ministry of Science and Technology (MOST), Government of the Bangladesh research fund (project ref. no. :39.009.006.01.00.049.2013-2014/MEDE'S-54/235/1(4) and (project ref. no.: 39.009.002.01.00.053.2014-2015/ Phys-275/379). We are deeply thankful to the Chairman, BCSIR, Dhaka, Bangladesh for providing the ${ }^{1} \mathrm{H}-\mathrm{NMR}$ spectra.

\section{References}

1. M.E.Taylor, K. Drickamer, Introduction to Glycobiology, Third edition, Oxford University Press, NY, USA (2011).

2. A. Varki, R.D. Cummings, J.D. Esko, H.H. Freeze, P. Stanley, Essentials of Glycobiology, Second edition, Cold Spring Harbor Laboratory Press, Cold Spring, Harbor, NY, USA (2009).

3. J.J. Willard, J.S. Brimacombe, R.P. Brueton, The synthesis of 3-O-[(benzylthio)carbonyl]- $\beta-D$ glucopyranose and methyl 2,4,6-tri-O- and $\beta$-Dglucopyranosides, Canad. J. Chem., 42 (1964) 2560.

4. D. Wagner, J.P.H. Verheyden, J.G. Moffatt, Preparation and synthetic utility of some organotin derivatives of nucleosides, J. Org. Chem., 39 (1974) 24.

5. R.M. Munavu, H.H. Szmant, Selective formation of 2 esters of some methyl-D-hexopyranosides via dibutylstannylene derivatives, J. Org. Chem., 41 (1976) 1832.

6. H. Ishii, M. Nakamura, S. Seo, K. Tori, T. Tozoyo, Y. Yoshimura, Isolation, characterization and nuclear magnetic spectra of new saponins from the roots of Bupleurum falcatum L, Chem. Pharm. Bull., 28 (1980) 2367.

7. Y. Tsuda, E. Haque, Regioselective introduction of p-coumaroyl group to -L-arabinopyranosides, Total synthesis of inundoside- $G$ and inundoside-D1, Chem. Pharm. Bull., 31 (1983) 1437.

8. A.K.M.S. Kabir, P. Dutta, M.N. Anwar, Synthesis of some new derivatives of D-mannose, Chittagong Univ. J. Sci., 29 (2005) 01.

9. D.K. Byarugaba, Antimicrobial resistance in developing countries and responsible risk factors, Int. J. Antimicrob. Agents, 24 (2004) 105.

10. S.J. Projan, D.M. Shlaes, Antibacterial drug discovery: is it all downhill from here, Clin. Microbiol. Infect, 10 (2004) 18.

11. J.J. Barker, Antibacterial drug discovery and structure-based design, Drug Discov. Today, 11 (2006) 391.

12. M.A. Bakht, M.S. Yar, S.G. Abdel-Hamid, S.I. Al Qasoumi, S. Abdul, Molecular properties prediction, synthesis and antimicrobial activity of some newer oxadiazole derivatives, Eur. J. Med. Chem., 45 (2010) 5862.
13. R.S. Kumar, A.I. Adhulla, A. J.A. Nasser, J. Selvin, Synthesis and antimicrobial activity of a new series of 1,4-dihydropyridine derivatives, J. Seb. Chem. Soc., 76 (2011) 01.

14. H. Singh, K.N. Shukla, R. Dwivedi, L.D.S. Yadav, Cycloaddition of 4-amino-3-mercapto-1,2,4-triazole to heterocumulenes and antifungal activity of the resulting 1,2,4-triazolo [3,4-c]-1,2-dithia-4,5-diazines, J. Agric. Food Chem., 38 (1990) 1483.

15. R. Gupta, S. Paul, A.K. Gupta, P.L. Kachroo, S. Bani, Synthesis and biological activities of some 2-substituted phenyl-3-(3-alkyl/aryl-5,6-dihydro-striazolo[3,4-b][1,3,4]thiazolo-6-yl)-indoles, Ind. J. Chem., 36 (1997) 707.

16. M.M. Ghorab, Z.H. Ismail, S.M.A. Gaward, A.A. Aziem, Antimicrobial activity of amino acid, imidazole and sulfonamide derivatives of pyrazolo[3,4-d]pyrimidine, Heteroatom Chem., 15 (2004) 57.

17. S.L. Gaonkar, K.M.L. Rai, B. Prabhuswamy, Synthesis and antimicrobial studies of a new series of 2- $\{4$-[2-(5-ethylpyridin-2-yl)ethoxy]phenyl\}-5substituted-1,3,4-oxadiazoles, Eur. J. Med. Chem., 41 (2006) 841.

18. Z.A. Kaplancikli, M.D. Altintop, G. Turan-Zitouni, A. Ozdemir, R. Ozic, G. Akalin, Synthesis, antimicrobial activity and cytotoxicity of novel oxadiazole derivatives, J. Enzyme Inhib. Med. Chem., 27 (2012) 51.

19. Q. Zheng, X. Zhang, X. Ying, K. Cheng, Q. Jiao, H. Zhu, Synthesis, biological evaluation, and molecular docking studies of 2 -chloropyridine derivatives possessing 1,3,4-oxadiazole moiety as potential antitumor agents, Bioorg. Med. Chem., 18 (2010) 7836.

20. S. Dash, B.A. Kumar, J. Sing, B.C. Maiti, T.K. Maity, Synthesis of some novel 3,5-disubstituted 1,3,4-oxadiazole derivatives and anticancer activity on EAC animal model, Med. Chem. Res., 20 (2011) 1206.

21. M. A. Ali, M. Shaharyar, Oxadiazole mannich bases: Synthesis and antimycobacterial activity, Bioorg. Med. Chem. Lett., 17 (2007) 3314.

22. R.S. Kumar, S. Arunachalam, V.S. Periasamy, C.P. Preethy, A. Riyasdeen, M.A. Akbarsha, DNA binding and biological studies of some novel water-soluble polymer-copper(II)-phenanthroline complexes, Eur. J. Med. Chem., 45 (2010) 2082.

23. K. Manjunatha, B. Poojary, P.L. Lobo, J. Fernandes, N.S. Kumari, Synthesis and biological evaluation of some 1,3,4-oxadiazole derivatives, Eur. J. Med. Chem., 45 (2010) 5225.

24. G.C. Ramaprasad, B. Kalluraya, B.S. Kumar, R.K. Hunnur, Synthesis and biological property of some novel 1,3,4-oxadiazoles, Eur. J. Med. Chem., 45 (2010) 4587.

25. A.A. El-Emam, O.A. Al-Deeb, M. Al-Omar, J. Lehmann, Synthesis, antimicrobial, and anti-HIV-1 activity of certain 5-(1-adamantyl)-2-substituted thio-1,3,4oxadiazoles and 5-(1-adamantyl)-3-substituted aminomethyl-1,3,4-oxadiazoline-2-thiones, Bioorg. Med. Chem., 12 (2004) 5107.

26. S.M.A. Kawsar, M.O. Faruk, M.S. Rahman, Y. Fujii, Y. Ozeki, Regioselective Synthesis, Characterization and Antimicrobial Activities of Some New Monosaccharide Derivatives, Sci. Pharm., 82 (2014) 01. 
27. S.M.A. Kawsar, T. Hasan, S.A. Chowdhury, M.M. Islam, M.K. Hossain, M.A. Manchur, Synthesis, Spectroscopic Characterization and in vitro Antibacterial Screening of some D-Glucose Derivatives, Int. J. Pure Appl. Chem., 8 (2013a) 125.

28. S.M.A. Kawsar, A.K.M.S. Kabir, M.M.R. Bhuiyan, J. Ferdous, M.S. Rahman, Synthesis, characterization and microbial screening of some new methyl 4,6-0-(4-methoxybenzylidene)-a-D-glucopyranoside derivatives, J. Bang. Acad. Sci., 37 (2013b) 145.

29. S.M.A. Kawsar, A.K.M.S. Kabir, M.M.R. Bhuiyan, M.M. Manik, Synthesis and characterization of methyl 4,6-0-benzylidene-a-D-glucopyranoside derivatives, J. Bang. Chem. Soc., 25 (2012a) 101.

30. S.M.A. Kawsar, A.K.M.S. Kabir, M.M.R. Bhuiyan, A. Siddiqa, M.N. Anwar, Synthesis, spectral and antimicrobial screening studies of some acylated D-glucose derivatives, Rajiv Gandhi Univ. Health Sci. (RGUHS) J. Pharm. Sci., 2 (2012b) 107.

31. S.M.A. Kawsar, A.K.M.S. Kabir, M.M. Manik, M.K. Hossain, M.N. Anwar, Antibacterial and mycelial growth inhibition of some acylated derivatives of D-glucopyranoside, Int. J. Biosci., 2 (2012c) 66.

32. A.K.M.S. Kabir, S.M.A. Kawsar, M.M.R. Bhuiyan, M.S. Rahman, M.E. Chowdhury, Antimicrobial screening studies of some derivatives of methyl a-Dglucopyranoside, Pak. J. Sci. Ind. Res., 52 (2009) 138.

33. A.K.M.S. Kabir, S.M.A. Kawsar, M.M.R. Bhuiyan, M.S Rahman, B. Banu, Biological evaluation of some octanoyl derivatives of methyl 4,6-0-cyclohexylidenea-D-glucopyranoside, Chittagong Univ. J. Biol. Sci., 3 (2008) 53.
34. A.K.M.S. Kabir, S.M.A. Kawsar, M.M.R. Bhuiyan, M.R. Islam, M.S. Rahman, Biological evaluation of some mannopyranoside derivatives, Bull. Pure Appl. Sci., 23 (2004) 83.

35. A.K.M.S. Kabir, P. Dutta, M.N. Anwar, Antimicrobial evaluation of some decanoyl derivatives of methyl $\alpha$-D-glucopyranoside, Int. J. Agric. Biol., 7 (2005b) 760 .

36. A.K.M.S. Kabir, S.M.A. Kawsar, M.M.R. Bhuiyan, M.S. Rahman, B. Banu, Biological evaluation of some octanoyl derivatives of methyl 4,6-0-cyclohexylidene$\alpha$-D-glucopyranoside, Chittagong. Univ. J. Sci., 2 (2007) 81.

37. A.W. Bauer, W.M.M. Kirby, J.C. Sherris, M. Turck, Antibiotic susceptibility testing by a standardized single disc method, Am. J. Clin. Pathol., 45 (1966) 439.

38. M.A.T. Miah, H.U. Ahmed, N.R. Sharma, A. Ali, S.A. Miah, Antifungal activity of some plant extracts, Bang. J. Bot., 19 (1990) 05.

39. R.K. Grover, J.D. Moore, Toximetric studies of fungicides against the brown rot organisms Sclerotinia flucticola and S. laxa, Phytopathology, 52 (1962) 876.

40. A.K.M.S. Kabir, P. Dutta, M.N. Anwar, Antibacterial and antifungal Evaluation of some Derivatives of methyl a-D-mannopyranoside, Int. J. Agr. Biol., 7 (2005) 756. 